\title{
Interpretazioni geofisiche della prima serie di triangolazioni eseguite presso la diga di Pieve di Cadore
}

\author{
M. C. SPADEA
}

\section{PARTE PRIMA}

\section{DULATAZIONE}

Fra le deformazioni che si possono verificare in un corpo solido elastico, vi è quella che si manifesta sotto forma di variazione di volume e che è nota con il nome di dilatazione. Ci limiteremo ad una esposizione sommaria.

Siano $\delta x, \delta y, \delta z$ gli spigoli di un parallelepipedo infinitesimo, riferito ad un sistema $x, y, z$, di assi triortogonali. Se $u, v, w$, sono le componenti dello spostamento subito dal vertice di coordinate $x, y, z$, e se $u, v$, $w$, sono funzioni di $x, y, z$, (e del tempo), $\frac{\partial u}{\partial x}, \frac{\partial v}{\partial y}, \frac{\partial v}{\partial z}$ esprimeranno le variazioni degli spostamenti, lungo i rispettivi spigoli, per unità di lunghezza.

Prima della deformazione il volume del parallelepipedo è dato da:

$$
V=\delta x \cdot \delta y \cdot \delta z
$$

In seguito alla deformazione, gli spigoli subiscono una variazione di lunghezza. Di conseguenza, il volume del parallelepipedo diverrà:

$$
\begin{aligned}
& V+\delta V= \\
& \quad=\delta x \delta y \delta z\left(1+\frac{\partial u}{\partial x}\right)\left(1+\frac{\partial v}{\partial y}\right)\left(1+\frac{\partial w}{\partial z}\right)
\end{aligned}
$$

Trascurando gli infinitesimi di ordine superiore al primo $\left(\frac{\partial u}{\partial x} \cdot \frac{\partial v}{\partial y}, \ldots\right)$ avremo:

Fatto

$$
\delta V=V\left(\frac{\partial u}{\partial x}+\frac{\partial v}{\partial y}+\frac{\partial w}{\partial z}\right)
$$

$$
\Delta=\frac{v w}{\partial x}+\frac{v u}{\partial y}+\frac{v w}{\partial z}
$$

consegue

$$
\Delta=\frac{\delta V}{V}
$$

$\Delta$ è detta dilatazione cubica, ed esprime la variazione dell'unità di volume sotto l'azione della forza deformante. Essa è data dalla somma degli allungamenti unitarii, relativi ad una qualsiasi terna di direzioni trirettangole.

Nel piano, $\Delta$ esprimerà la variazione di superficie e con riferimento al piano $x, y,-$ assumerà la forma

$$
\Delta=\frac{\partial u}{\partial x}+\frac{\partial v}{\partial y} .
$$

DISTORSIONE.

Oltre a variazioni di lunghezza, la forza perturbante puó determinare variazioni infinitesime nella direzione dei segmenti, che costituiscono gli spigoli del parallelepipedo considerato.

Siano $M A, M C$ gli spigoli di una faccia del parallelepipedo paralleli agli assi $x, z$, rispettivamente. Si prova che, se indichiamo con $2 \sigma_{2}$ la variazione infinitesima subita dall'angolo formato dai due spigoli - prima della deformazione uguale a $\frac{\pi}{2}-$, si ha

$$
\cos \left(\frac{\pi}{2}-\hat{z} v_{2}\right)=\frac{\partial u}{\partial z}+\frac{\partial w}{\partial x} .
$$

Due relazioni analoghe valgono per le facce parallele ai piani $z y$ e $x y$ rispettivamente; sicchè, tenuto conto che le variazioni sono infinitesime, si puó scrivere:

$$
\begin{aligned}
& \sigma_{1}=\frac{1}{2}\left(\frac{\partial w}{\partial y}+\frac{\partial v}{\partial z}\right) \\
& \sigma_{2}=\frac{1}{2}\left(\frac{\partial u}{\partial z}+\frac{\partial w}{\partial x}\right) \\
& \sigma_{3}=\frac{1}{2}\left(\frac{\partial v}{\partial x}+\frac{\partial u}{\partial y}\right)
\end{aligned}
$$


relazioni che esprimono le variazioni angolari degli spigoli del parallelepipedo, per effetto della deformazione.

Per un'interpretazione fisica della [3], si puó considerare una particolare sollecitazione elastica, che non provochi variazioni di sorta nelle grandezze in giuoco, escluse due. Siano queste p. es. $\frac{\partial u}{\partial z}$ e $\frac{\partial w}{\partial x}$. Sarà allora $\sigma_{1}=\sigma_{3}=0, \sigma_{2} \neq 0$. La deformazione interessa solo quindi le facce parallele al piano $x z$.

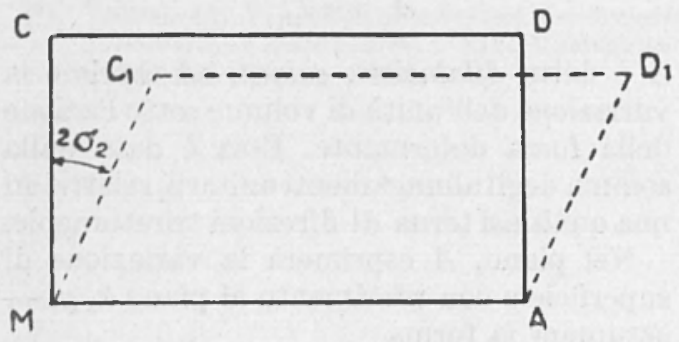

Fig. 1

Poichè qui interessano solo le variazioni relative, con riferimento alla fig. 1, possiamo ritenere che i punti $M, A$ restino fissi, sicchè $M O_{1} D_{1} A$ rappresenti la faccia $M C D A$ dopo la deformazione. Per la ipotesi fatta, $M C D A$ e $M C_{1} D_{1} A$ sono complanari.

La sollecitazione elastica considerata provoca quindi uno scorrimento dello spigolo $C D$ in $C_{1} D_{1}$, accompagnato da una deviazione angolare di $M C$ rispetto ad $M A$ espressa dalla grandezza $2 \sigma_{2}$.

Una simile deformazione è detta scorrimento o distorsione ("glissemant) dei Francesi o "Schiebungen " dei Tedeschi, "shear" degli Inglesi): essa rappresenta la variazione dell'angolo, inizialmente retto, della coppie di elementi lineari paralleli agli assi $x, z$. In altre parole, in forza di detta deformazione, la proiezione di $C M$ su $M A$, inizialmente nulla, nel passaggio dall'una all'altra configurazione, subisce uno scorrimento, dipendente da $\sigma_{2}$.

Poichè $\sigma_{2}$ è grandezza infinitesima, la deformazione considerata lascia immutate le aree.

Estendendo le stesse considerazioni alle altre facce, ne segue che il volume rimane invariato, come conseguenza dell'invarianza delle aree facciali e della lunghezza degli spigoh del parallelepipedo.

La distorsione è quindi caratterizzata da

$$
\Delta=0 \text {. }
$$

\section{ROTAZIONE.}

Si suole indicare con questo nome la deformazione caratterizzata dalle seguenti espressioni:

$$
\begin{aligned}
& \xi=\frac{1}{2}\left(\frac{\partial w}{\partial y}-\frac{\partial v}{\partial z}\right) \\
& \eta=\frac{1}{2}\left(\frac{\partial u}{\partial z}-\frac{\partial w}{\partial x}\right) \\
& \zeta=\frac{1}{2}\left(\frac{\partial v}{\partial x}-\frac{\partial u}{\partial y}\right)
\end{aligned}
$$

dove $u, v, w$, indicano, come in precedenza, le componenti dello spostamento subito da un punto di un corpo elastico sottoposto a deformazione.

L'interpretazione fisica della [4] giustifica la denominazione della deformazione che esse rappresentano.

Mi limito alla $2^{\text {a }}$ delle [4].

Sia $P$ un punto di coordinate $x, y, z$, del corpo solido considerato e proiettiamolo ortogonalmente sull'asse $y$.

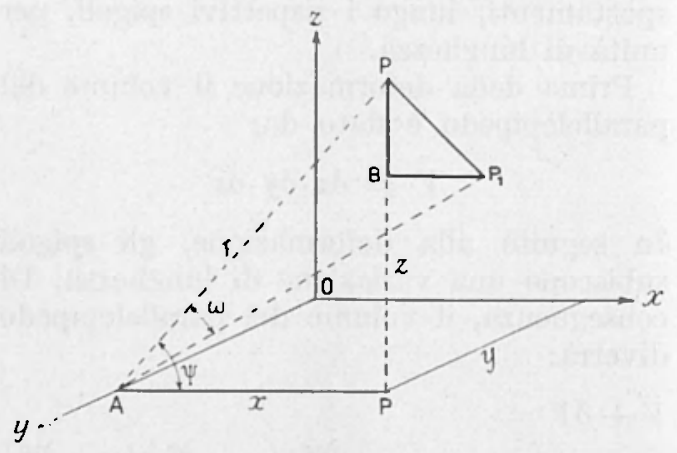

Fig. 2

Chiamando $r$ la distanza $A P$ e $\psi$ l'angolo che la retta $A P$ fa con il piano $x y$, avremo

$$
x=r \cos \psi \quad, \quad z=r \sin \psi
$$

Supponendo che tutto il corpo solido, contenente $P$, subisca una rotazione intorno ad $y$, indico con $\omega$ l'angolo di rotazione, che porta $P$ in $P_{1}$. Essendo l'angolo infinitesimo, si puó confondere l'archetto $P P_{1}$ 
con il corrispondente tratto di tangente in $P$. Si ha

$$
P P_{1}=r \omega \text {. }
$$

Le componenti dello spostamento rispetto ad $x$ e a $z$, saranno

$$
u=B P_{1}, w=B P ;
$$

pertanto

$$
u-r \omega \sin \psi \quad, \quad w=-r \omega \cos \psi
$$

e quindi per la [5],

$$
u=z \omega, \quad w=-x \omega .
$$

Ne consegue

$$
\omega=\frac{1}{2}\left(\frac{\partial u}{\partial z}-\frac{\partial w}{\partial x}\right),
$$

cloe:

$$
\omega=\eta
$$

La $\eta$ esprime perciò la rotazione infinitesima subita dalla particella considerata nel piano $z x$, intorno all'asse $y$, lungo il quale si propaga il movimento determinato dalla sollecitazione elastica. Analoga conclusione per $\xi$ e $\zeta$.

Le [-t] costituiscono quindi altrettante rotazioni infinitesime, subite dal punto considerato intorno agli assi.

Le [1]-[2] e le [4] hanno fondamentale importanza in tutta la teoria dell'elasticità; esse entrano nelle equazioni di propagazione dei piccoli moti in un corpo elastico e caratterizzano rispettivamente le onde longitudinali e trasversali in un corpo elastico.

\section{DEFORMAZIONI PRINCIPALI E LORO DIREZIONI.}

In un corpo elastico, per ogni punto $x, y, z$, passa una superficie quadrica della famiglia

$\frac{\partial u}{\partial x} x^{2}+\frac{\partial v}{\partial y} y^{2}+\frac{\partial w}{\partial z} z^{2}+\left(\frac{\partial w}{\partial y}+\frac{\partial v}{\partial z}\right) y z+$ $+\left(\frac{\partial u}{\partial z}+\frac{\partial w}{\partial x}\right) z w+\left(\frac{\partial v}{\partial x}+\frac{\partial u}{\partial y}\right) x y=$ cost.,

detta quadrica della deformazione. Sono note le proprietà di cui gode tale superficie. Se per es. la quadrica è un ellissoide, tutte le linee uscenti dal punto $(x, y, z)$, sono tese, oppure tutte contratte.

Nello stato indeformato, le direzioni delle linee per le quali l'estensione è un massimo o un minimo, o è stazionaria — senza essere un vero massimo o un vero minimo sono gli assi principali delle quadriche; e sono anche gli assi principali della deformazione.

Le direzioni degli assi principali della deformazione possono essere determinate nel seguente modo.

Siano, $l, m, n$, i coseni direttori di uno di questi assi, si ha allora

$$
\begin{aligned}
& \frac{2 \frac{\partial u}{\partial x} l+\left(\frac{\partial v}{\partial x}+\frac{\partial u}{\partial y}\right) m+\left(\frac{\partial u}{\partial z}+\frac{\partial w}{\partial x}\right) \hat{l}}{l}= \\
& =\frac{\left(\frac{\partial v}{\partial x}+\frac{\partial u}{\partial y}\right) l+2 \frac{\partial v}{\partial y} m+\left(\frac{\partial w}{\partial y}+\frac{\partial v}{\partial z}\right) n}{m}= \\
& =\frac{\left(\frac{\partial u}{\partial z}+\frac{\partial w}{\partial x}\right) l+\left(\frac{\partial w}{\partial y}+\frac{\partial v}{\partial z}\right) m+2 \frac{\partial w}{\partial z} n}{n} .
\end{aligned}
$$

Se $\gamma$ indica l'una o l'altra di queste quantità i tre possibili valori di $\gamma$ sono le radici dell'equazione

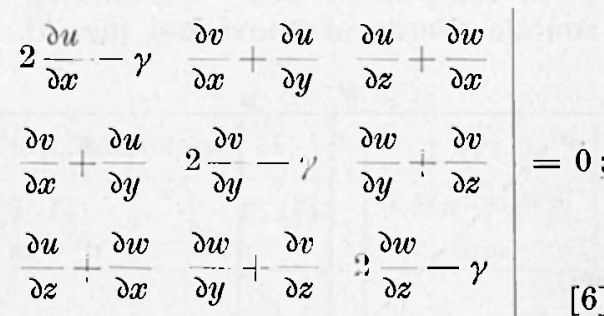

queste radici sono reali e sono i valori delle estensioni principali $\gamma_{1}, \gamma_{2}, \gamma_{3}$.

Considero la deformazione sul piano orizzontale. Con riferimento ad un arbitrario sistema di coordinate $x, y$, siano $u, v$, le componenti dello spostamento di un punto trigonometrico.

Per le [6], i valori delle deformazioni principali saranno dati dalle radici dell'equazione

$$
\begin{array}{cc}
2 \frac{\partial u}{\partial x}-\gamma & \frac{\partial v}{\partial x}+\frac{\partial u}{\partial y} \\
\frac{\partial v}{\partial x}+\frac{\partial u}{\partial y} & 2 \frac{\partial v}{\partial y}-\gamma
\end{array} \mid=0 .
$$

Se $\vartheta$ indica l'angolo che gli assi delle deformazioni principali formano con l'asse 
$x$, consegue

$$
\operatorname{tang} \vartheta=-\frac{2 \frac{\partial u}{\partial x}-\gamma}{\frac{\partial v}{\partial x}+\frac{\partial u}{\partial y}}
$$

Dalla [7] si trae agevolmente

$$
\begin{gathered}
\gamma_{2}{ }^{1}=\frac{\partial u}{\partial x}+\frac{\partial v}{\partial y} \pm \\
\pm \sqrt{\left(\frac{\partial u}{\partial x}-\frac{\partial v}{\partial y}\right)^{2}+\left(\frac{\partial v}{\partial x}+\frac{\partial u}{\partial y}\right)^{2}}
\end{gathered}
$$

Per la [8], si ha infine

$$
\vartheta_{1,2}=\operatorname{arctg} \frac{\frac{\partial v}{\partial y}-\frac{\partial u}{\partial x}-\sqrt{\left(\frac{\partial u}{\partial x}-\frac{\partial v}{\partial y}\right)^{2}+\left(\frac{\partial v}{\partial x}+\frac{\partial u}{\partial y}\right)^{2}}}{\frac{\partial v}{\partial x} ; \frac{\partial u}{\partial y}}
$$

\section{MASSIMA DISTORSIONE.}

Si consideri un nuovo sistema di coordinate, i cui assi $x^{\prime}, y^{\prime}$, risultino di un angolo $v$ rispetto agli assi $x, y$. Indico con $u^{\prime}$, $v^{\prime}$ le componenti dello spostamento orizzontale riferite ai nuovi assi (fig. 3 ).

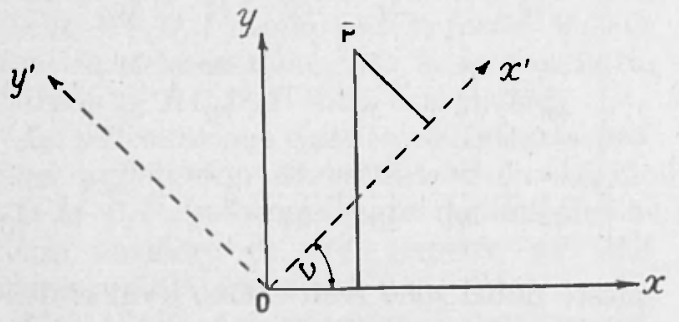

Fig, 3

Sarà allora — con riferimento alle [7] —

$$
\begin{gathered}
2 \sigma=\frac{\partial v^{\prime}}{\partial x^{\prime}}+\frac{\partial u^{\prime}}{\partial y^{\prime}}= \\
=\left(\frac{\partial v}{\partial x}+\frac{\partial u}{\partial y}\right) \cos 2 v+\left(\frac{\partial v}{\partial y}-\frac{\partial u}{\partial w}\right) \sin 2 v .
\end{gathered}
$$

Il valore che rende massimo $2 \sigma$ si deduce da $\frac{\partial 2 \sigma}{\partial v}=0$.

Questa condizione si traduce nelle relazione:

$$
\left[\underset{2 \sigma \rightarrow \max }{\operatorname{tang} 2 v]^{2}}=\left(\frac{\partial v}{\partial y}-\frac{\partial u}{\partial x}\right) /\left(\frac{\partial u}{\partial y}+\frac{\partial v}{\partial x}\right)\right.
$$

in forza della quale la [11] diviene

$$
\begin{gathered}
2 \sigma=\left[\left(\frac{\partial u}{\partial y}+\frac{\partial v}{\partial x}\right)^{2}+\left(\frac{\partial v}{\partial y}-\frac{\partial u}{\partial x}\right)^{2}\right] \cos 2 v \\
/\left(\frac{\partial u}{\partial y}+\frac{\partial v}{\partial x}\right)
\end{gathered}
$$

Consegue pertanto

$$
\begin{gathered}
\sigma_{\max }-\sqrt{\left(\frac{\partial v}{\partial x}+\frac{\partial u}{\partial y}\right)^{2}+\left(\frac{\partial v}{\partial y}-\frac{\partial u}{\partial x}\right)^{2}}= \\
=\frac{1}{2}\left(\gamma_{1}-\gamma_{2}\right) .
\end{gathered}
$$

La $\sigma_{\max }$ risulta indipendente dalla scelta degli assi coordinati come del resto la distorsione e la deformazione principale.

Le formule su riportate permettono la determinazione degli elementi principali che caratterizzano elasticamente una regione. La loro conseguenza presenta un interesse notevole, sia dal punto di vista puramente sismico - per ciò che si riferisce a zone di elevata sismicità — sia con riferimento alla stabilità di ristrette zone, l'equilibrio delle quali sia stato comunque turbato da profondi scavi e da pesanti costruzioni.

Le grandi dighe di sbarramento rientrano, evidentemente, in quest'ultimo caso.

Le triangolazioni che, periodicamente, vengono eseguite nei luoghi ove sorgono queste grandi dighe, alla luce dei procedimenti sopra riassunti vengono ad assumere un significato che va ben oltre al semplice interesse geodetico. La loro elaborazione in campo geofisico permette infatti il conseguimento di preziosi elementi, atti a mettere in evidenza l'andamento della variazione di quelle caratteristiche elastiche del terreno, che sono peculiari per la stabilità dei manufatti in esso ospitati.

Mi sono pertanto proposta di farne un'applicazione ad alcune delle triangolazioni che, dal 1950, vengono periodicamente eseguite presso la diga di Pieve di Cadore.

ELABORAZIONE GEOFISICA DI ALCUNE TRIANGOLAZIONI ESEGUTTE PRESSO LA DIGA DI Pieve di Cadore.

Si consideri un triangolo i cui vertici siano di coordinate $\left(x_{1}, y_{1}\right),\left(x_{2}, y_{2}\right),\left(x_{3}, y_{3}\right)$. Gli spostamenti orizzontali osservati di 
questi punti siano $\left(u_{1}, v_{1}\right),\left(u_{2}, v_{2}\right),\left(u_{3}, v_{3}\right)$ rispettivamente.

Nell'interno del triangolo, i valori di $u$ e $v$, in prima approssimazione, possono essere rappresentate dalle espressioni $u=a x+b y+c$, $v=a^{\prime} x+b^{\prime} y+c^{\prime}$, soggette alle condizioni

$$
\begin{array}{ll}
u_{1}=a x_{1}+b y_{1}+c & v_{1}=a^{\prime} x_{1}+b^{\prime} y_{1}+c^{\prime} \\
u_{2}=a x_{2}+b y_{2}+c & v_{2}=a^{\prime} x_{2}+b^{\prime} y_{2}+c^{\prime} \\
u_{3}=a x_{3}+b y_{3}+c & v_{3}=a^{\prime} x_{3}+b^{\prime} y_{3}+c^{\prime} .
\end{array}
$$

Si ha:

$$
\begin{aligned}
& u_{2}-u_{1}=a\left(x_{2}-x_{1}\right)+b\left(y_{2}-y_{1}\right) \\
& u_{3}-u_{1}=a\left(x_{3}-x_{1}\right)+b\left(y_{3}-y_{1}\right),
\end{aligned}
$$

ed espressioni analoghe per $v_{2}-v_{1}, v_{3}-v_{1}$. Consegue

$$
\begin{aligned}
& \frac{\partial u}{\partial x}=\frac{\left(y_{3}-y_{1}\right)\left(u_{2}-u_{1}\right)-\left(y_{2}-y_{1}\right)\left(u_{3}-u_{1}\right)}{D} \\
& \frac{\partial u}{\partial y}=\frac{\left(x_{2}-x_{1}\right)\left(u_{3}-u_{1}\right)-\left(x_{3}-x_{1}\right)\left(u_{2}-u_{1}\right)}{D} \\
& \frac{\partial v}{\partial x}=\frac{\left(y_{3}-y_{1}\right)\left(v_{2}-v_{1}\right)-\left(y_{2}-y_{1}\right)\left(v_{3}-v_{1}\right)}{D} \\
& \frac{\partial v}{\partial y}=\frac{\left(x_{2}-x_{1}\right)\left(v_{3}-v_{1}\right)-\left(x_{3}-x_{1}\right)\left(v_{2}-v_{1}\right)}{D}
\end{aligned}
$$

dove

$$
D=\left(x_{2}-x_{1}\right)\left(y_{3}-y_{1}\right)-\left(x_{3}-x_{1}\right)\left(y_{2}-y_{1}\right) .
$$

Nelle Tabelle da 1 a 7 sono riportate le coordinate dei vertici per le varie triangolazioni - dalla II all'VIII - eseguite presso la diga di Pieve di Cadore, dall'Agosto 1949 al Giugno 1952.

L'ultima colonna di ogni tavola dà gli spostamenti relativi dei vertici, osservati tra una triangolazione e la precedente.

Nelle Tabelle da 8 a 15 sono riportati i valori di $\frac{\partial u}{\partial x}, \frac{\partial u}{\partial y}, \frac{\partial v}{\partial x}, \frac{\partial v}{\partial y}$, calcolati valendosi delle formule 13 e 14 .

Nelle colonne successive sono riportati i valori della dilatazione $\Delta$ [formula 2],

\begin{tabular}{|c|c|c|c|c|c|c|}
\hline \multirow{2}{*}{ VERTICI } & \multicolumn{2}{|c|}{ Triangolazione I } & \multicolumn{2}{|c|}{ Triangolazione II } & \multirow{2}{*}{\multicolumn{2}{|c|}{$\begin{array}{c}T(I I-I) \\
\sqrt{\Delta^{2} x+\Delta^{2} y} \\
\mathrm{~mm}\end{array}$}} \\
\hline & $\underset{\mathrm{m}}{X(T \cdot I)}$ & $\underset{\mathrm{m}}{Y(T \cdot I)}$ & $\underset{\mathrm{m}}{X(T \cdot I I)}$ & $\underset{\mathrm{m}}{Y(T \cdot I I)}$ & & \\
\hline $13 \mathrm{M}$ & $1.000,0000$ & $1.000,0000$ & $1.000,0000$ & $1.000,0000$ & & - \\
\hline $11 M$ & 784,3211 & $1.462,7053$ & 784,3212 & $1.462,7049$ & 4 & - $10^{-4}$ \\
\hline 6 & $1.053,8529$ & $1.374,3456$ & $1.053,8552$ & $1.374,3426$ & 38 & - $10^{-4}$ \\
\hline 7 & 802,4214 & $1.593,2983$ & 802,4229 & $1.593,3043$ & 62 & $\cdot 10^{-4}$ \\
\hline 8 & 1. 194,8979 & $1.828,7578$ & $1.194,9145$ & $1.828,7646$ & 179 & . $10^{-4}$ \\
\hline 9 & 760,4946 & $1.799,6340$ & 760,4956 & $1.799,6407$ & 68 & $\cdot 10^{-1}$ \\
\hline 10 & $1.114,9322$ & $2.075,7743$ & $1.144,9439$ & $2.075,7909$ & 203 & $\cdot 10^{-4}$ \\
\hline 11 & 745,1598 & $2.008,5224$ & 745,1652 & $2.008,5409$ & 193 & . $10^{-4}$ \\
\hline 12 & 808,4623 & $2.389,4288$ & 808,4798 & $2.389,4602$ & 359 & . $10^{-4}$ \\
\hline 13 & $1.353,8743$ & 2.433 .5338 & $1.353,9016$ & $2.433,5697$ & 511 & $\cdot 10^{-4}$ \\
\hline \multicolumn{2}{|c|}{$\begin{array}{l}\text { Triangolazione I } \\
\text { l5/VIII - } 7 \text { /XI } 1949\end{array}$} & \multicolumn{2}{|c|}{ Quota Bacino - $630 \div 640$} & & & \\
\hline \multicolumn{2}{|c|}{$\begin{array}{l}\text { TriangolazIONE II } \\
3-31 / \text { VIII } 1950\end{array}$} & \multicolumn{2}{|c|}{ Quota Bacino - Pieno } & & & \\
\hline
\end{tabular}
distorsione $\sigma$ [la $3^{\text {a }}$ delle formule 3], rotazione $\omega$ [la $3^{\mathrm{a}}$ delle formule 4], distorsione massima $\sigma_{M}$ [formula 12], delle deformazioni principali $\gamma_{1}, \gamma_{2}$ [formula 9], nonchè dell'angolo $\vartheta$ che gli assi delle deformazioni principali formano con l'asse $x$ [formula 8],

I numeri sono espressi in unità pari a $10^{-8} \mathrm{~cm}$.

Tabella 1 
Tabella 2

\begin{tabular}{|c|c|c|c|c|c|c|}
\hline \multirow{2}{*}{ Vertici } & \multicolumn{2}{|c|}{ Triafgolazione II } & \multicolumn{2}{|c|}{ Triangolazione III } & \multirow{2}{*}{\multicolumn{2}{|c|}{$\begin{array}{c}T(I I I-I I) \\
\sqrt{\Delta^{2} x+\Delta^{2} y} \\
\mathrm{~mm}\end{array}$}} \\
\hline & $\begin{array}{c}X(T \cdot I I) \\
\mathrm{m}\end{array}$ & $\begin{array}{c}Y(T \cdot I I) \\
\mathrm{m}\end{array}$ & $\underset{\mathrm{m}}{X}(T \cdot I I I)$ & $\underset{\mathrm{m}}{\mathrm{I}}(T \cdot I I I)$ & & \\
\hline $13 \mathrm{M}$ & $1.000,0000$ & $1.000,0000$ & 1. 000,0000 & 1. 000,0000 & & \\
\hline $11 \mathrm{M}$ & 784,3212 & $1.462,7049$ & 784,3213 & $1.462,7046$ & 3 & $10^{-1}$ \\
\hline 6 & $1.053,8552$ & $1.374,3426$ & 1. 053,8483 & $1.374,3447$ & 72 & $10^{-1}$ \\
\hline 7 & 802,4229 & $1.593,3043$ & 802,4212 & $1.593,2990$ & 56 & $10^{-4}$ \\
\hline 8 & $1.194,9145$ & $1.828,7646$ & 1. 194,8864 & $1.828,7564$ & 293 & $10^{-1}$ \\
\hline 9 & 760,4956 & 1. 799,6407 & 760,4954 & 1. 799,6259 & 148 & $10^{-4}$ \\
\hline 10 & $1.114,9439$ & $2.075,7909$ & $1.114,9195$ & $2.075,7693$ & 326 & $10^{-1}$ \\
\hline 11 & 745,1652 & $2.008,5409$ & 745,1628 & $2.008,5198$ & 212 & $10^{-4}$ \\
\hline 12 & 808,4798 & $2.389,4602$ & 808,4682 & $2.389,4230$ & 390 & $10^{-1}$ \\
\hline 13 & $1.353,9106$ & $2.433,5697$ & $1.353,8615$ & $2.433,5240$ & 671 & $10^{-1}$ \\
\hline \multicolumn{2}{|c|}{$\begin{array}{l}\text { TRIANGOLAZIONE II } \\
3 \cdot 31 / \text { VIII } 1950\end{array}$} & \multicolumn{2}{|c|}{ Quota Bacino - Pieno } & & & \\
\hline \multicolumn{2}{|c|}{$\begin{array}{l}\text { Triangolazione III } \\
29 / 11 \mathrm{I}-18 / \mathrm{IV} 1951\end{array}$} & \multicolumn{2}{|c|}{ Quota Bacino - 662,00 } & & & \\
\hline
\end{tabular}

Tabella 3

\begin{tabular}{|c|c|c|c|c|c|c|}
\hline \multirow{2}{*}{ VERTICI } & \multicolumn{2}{|c|}{ Triangolazione III } & \multicolumn{2}{|c|}{ Triangolazione IV } & \multirow{2}{*}{\multicolumn{2}{|c|}{$\frac{T(I V-I I I)}{\sqrt{\Delta^{2} x+\Delta^{2} y}}$}} \\
\hline & $\underset{\mathrm{m}}{X(T \cdot I I I)}$ & $\begin{array}{c}Y(T \cdot I I I) \\
\mathrm{m}\end{array}$ & $\begin{array}{c}X(T \cdot I V) \\
\mathrm{m}\end{array}$ & $\begin{array}{c}\mathrm{Y}(T \cdot I V) \\
\mathrm{m}\end{array}$ & & \\
\hline $13 \mathrm{M}$ & $1.000,0000$ & $1.000,0000$ & $1.000,0000$ & $1.000,0000$ & & \\
\hline $11 \mathrm{M}$ & 784,3213 & $1.462,7046$ & 784,3210 & $1.462,7057$ & 11 & $10^{-4}$ \\
\hline 6 & $1.053,8483$ & $1.374,3447$ & $1.053,8575$ & $1.374,3433$ & 93 & $10^{-1}$ \\
\hline 7 & 802,4212 & $1.593,2990$ & 802,4213 & $1.593,3041$ & 51 & $10^{-4}$ \\
\hline 8 & $1.194,8864$ & $1.828,7564$ & 1. 194,9072 & $1.828,7704$ & 251 & $10^{-4}$ \\
\hline 9 & 760,4954 & $1.799,6259$ & 760,4963 & 1. 799,6465 & 206 & $10^{-4}$ \\
\hline 10 & $1.114,9195$ & $2.075,7693$ & $1.114,9404$ & $2.075,7955$ & 337 & $10^{-1}$ \\
\hline 11 & 745,1628 & $2.008,5198$ & 745,1707 & $2.008,5469$ & 275 & $10^{-1}$ \\
\hline 12 & 808,4682 & $2.389,4230$ & 808,4751 & $2.389,4631$ & 407 & $10^{-4}$ \\
\hline 13 & $1.353,8615$ & $2.433,5240$ & 1. 353,9016 & $2.433,5696$ & 607 & $10^{-1}$ \\
\hline \multicolumn{2}{|c|}{$\begin{array}{l}\text { Triangolazione III } \\
29 / 111-18 / \text { IV } 1951\end{array}$} & \multicolumn{2}{|c|}{ Quota Bacino - 662,00} & & & \\
\hline \multicolumn{2}{|c|}{$\begin{array}{l}\text { TriangolazIONE IV } \\
21-31 / \mathrm{V} 1951\end{array}$} & \multicolumn{2}{|c|}{ Quota Bacino -- Vuoto } & & & \\
\hline
\end{tabular}


'Tabella 4

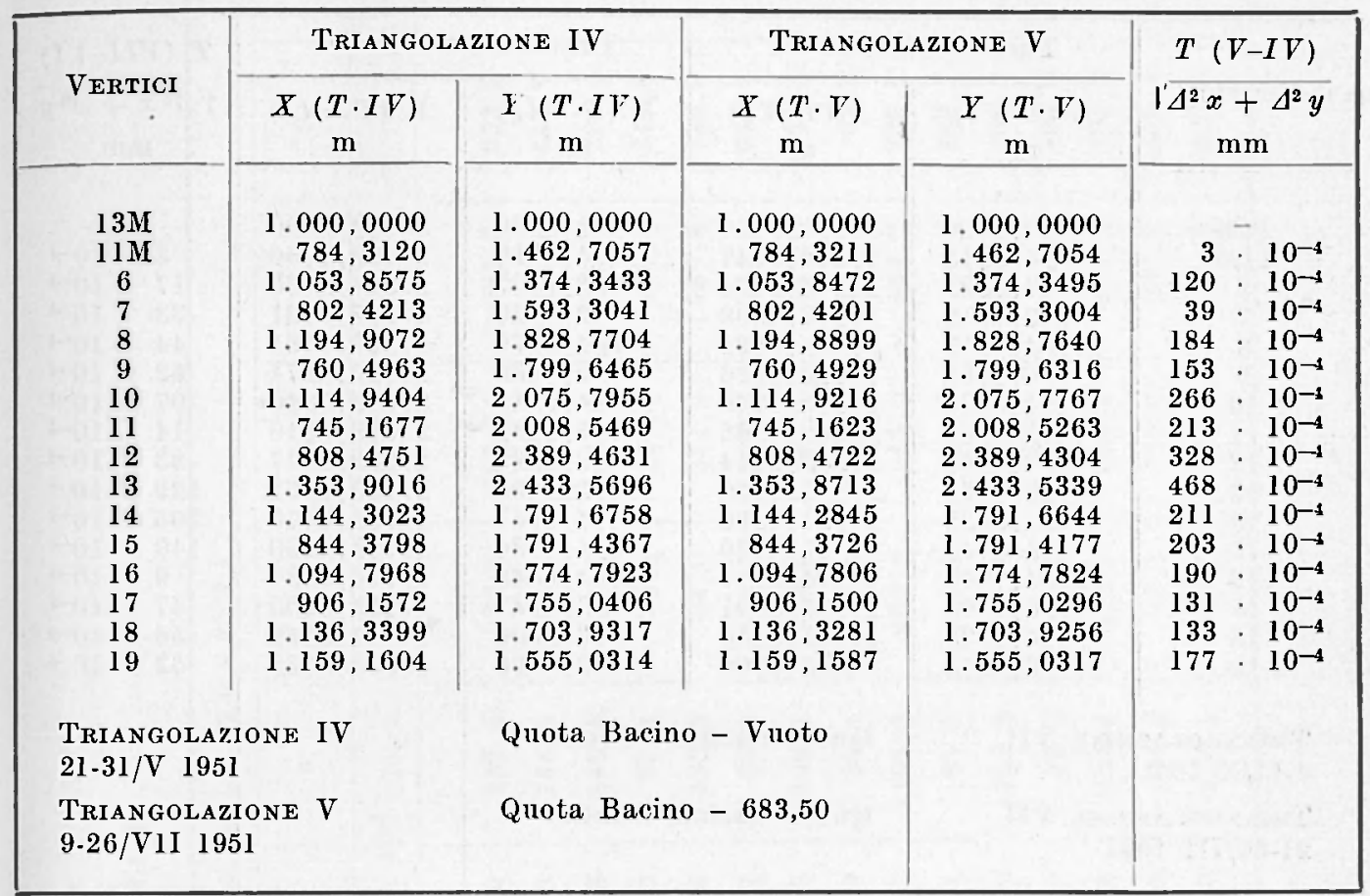

Tabella 5

\begin{tabular}{|c|c|c|c|c|c|}
\hline \multirow{2}{*}{ VERTICI } & \multicolumn{2}{|c|}{ Triangolazione V } & \multicolumn{2}{|c|}{ Triangolazione VI } & \multirow{2}{*}{$\begin{array}{c}T(V I-V) \\
\sqrt{\Delta^{2} x+\Delta^{2} y} \\
\text { mm }\end{array}$} \\
\hline & $\begin{array}{l}X(T \cdot V) \\
\mathrm{m}\end{array}$ & $\begin{array}{c}Y(T \cdot Y) \\
\mathrm{m}\end{array}$ & $\begin{array}{c}X(T \cdot V I) \\
\mathrm{m}\end{array}$ & $\begin{array}{c}Y(T \cdot V I) \\
\mathrm{m}\end{array}$ & \\
\hline $13 M$ & $1.000,0000$ & $1.000,0000$ & 1.000 .0000 & 1.0000000 & _- \\
\hline $11 \mathrm{M}$ & 784,3211 & $1.462,7054$ & 784,3213 & $1.462,7047$ & $.7 \cdot 10^{-4}$ \\
\hline 6 & $1.053,8472$ & $1.374,3495$ & $1.053,8521$ & 1. 374,3426 & $85 \cdot 10^{-4}$ \\
\hline 7 & 802,4201 & 1.593,3004 & 802,4219 & 1. 593,2998 & $19 \cdot 10^{-4}$ \\
\hline 8 & 1. 194,8899 & 1. 828,7640 & 1. 194,8959 & 1. 828,7621 & $63 \cdot 10^{-4}$ \\
\hline 9 & 760,4929 & 1. 799,6316 & 760,4952 & 1. 799,6326 & $24 \cdot 10^{-4}$ \\
\hline 10 & $1.114,4216$ & $2.075,7767$ & $1.114,9293$ & $2.075,7746$ & $80 \cdot 10^{-4}$ \\
\hline 11 & 745,1623 & $2.008,5263$ & 745,1631 & $2.008,5296$ & $34 \cdot 10^{-4}$ \\
\hline 12 & 808,4722 & $2.389,4304$ & 808,4753 & $2.389,4414$ & $144 \cdot 10^{-4}$ \\
\hline 13 & $1.353,8713$ & $2.433,5339$ & 1. 353,8955 & $2.433,5450$ & $266 \cdot 10^{-4}$ \\
\hline 14 & $1.144,2845$ & $1.791,6644$ & 1. 144,2888 & 1. 791,6596 & $65 \cdot 10^{-4}$ \\
\hline 15 & 844,3726 & $1.791,4177$ & 844,3727 & 1. 791,4150 & $27 \cdot 10^{-1}$ \\
\hline 16 & $1.094,7806$ & $1.774,7824$ & $1.094,7840$ & l. 774,7841 & $38 \cdot 10^{-4}$ \\
\hline 17 & 906,1500 & 1. 755,0296 & 906,1490 & 1. 755,0301 & $11 \cdot 10^{-4}$ \\
\hline 18 & $1.136,3281$ & 1. 703,9256 & $1.136,3275$ & 1. 703,9215 & $42 \cdot 10^{-4}$ \\
\hline 19 & $1.159,1587$ & $1.555,0317$ & $1.159,1552$ & $1.555,0306$ & $37 \cdot 10^{-4}$ \\
\hline \multicolumn{2}{|c|}{$\begin{array}{l}\text { Triangolazione V } \\
9-26 / \text { VII } 1951\end{array}$} & \multicolumn{2}{|c|}{ Quota Bacino - 683,50 } & & \\
\hline \multicolumn{2}{|c|}{$\begin{array}{l}\text { Trivngolazione VI } \\
4-11 / \mathrm{X} 1951\end{array}$} & \multicolumn{2}{|c|}{ Quota Bacino - Pieno } & & \\
\hline
\end{tabular}


Tabella 6

\begin{tabular}{|c|c|c|c|c|c|}
\hline \multirow{2}{*}{ VERTICI } & \multicolumn{2}{|c|}{ Triangolazione VI } & \multicolumn{2}{|c|}{ Triangolazione VII } & \multirow{2}{*}{$\begin{array}{c}T(V I I-V I) \\
\mathfrak{1}^{\prime} \Delta^{2} x+\Delta^{2} y \\
\mathrm{~mm}\end{array}$} \\
\hline & $\underset{\mathrm{m}}{X(T \cdot V I)}$ & $\underset{\mathrm{m}}{Y(T \cdot V I)}$ & $\underset{\mathrm{m}}{X(T \cdot V I I)}$ & $\begin{array}{c}Y(T . V I I) \\
\mathbf{m}\end{array}$ & \\
\hline $\begin{array}{c}13 \mathrm{M} \\
11 \mathrm{M} \\
6 \\
7 \\
8 \\
9 \\
10 \\
11 \\
12 \\
13 \\
14 \\
15 \\
16 \\
17 \\
18 \\
19\end{array}$ & $\begin{array}{r}1.000,0000 \\
784,3213 \\
1.053,8521 \\
802,4219 \\
1.194,8959 \\
760,4952 \\
1.114,9293 \\
745,1631 \\
808,4753 \\
1.353,8955 \\
1.144,2889 \\
844,3727 \\
1.094,7840 \\
906,1490 \\
1.136,3275 \\
1.159,1552\end{array}$ & $\begin{array}{l}1.000,0000 \\
1.462,7047 \\
1.374,3426 \\
1.593,2998 \\
1.828,7621 \\
1.799,6226 \\
2.075,7746 \\
2.008,5296 \\
2.389,4414 \\
2.433,5450 \\
1.791,6596 \\
1.791,4150 \\
1.774,7841 \\
1.755,0301 \\
1.703,9215 \\
1.555,3006\end{array}$ & $\begin{array}{r}1.000,0000 \\
784,3212 \\
1.053,8507 \\
802,4220 \\
1.194,8978 \\
760,4966 \\
1.114,9315 \\
745,1631 \\
808,4699 \\
1.353,8892 \\
1.144,2957 \\
844,3736 \\
1.094,7848 \\
906,1547 \\
1.136,3309 \\
1.159,1596\end{array}$ & $\begin{array}{l}1.000,0000 \\
1.462,7050 \\
1.374,3435 \\
1.593,3031 \\
1.828,7661 \\
1.799,6374 \\
2.075,7840 \\
2.008,5310 \\
2.389,4477 \\
2.433,5562 \\
1.791,6675 \\
1.791,4290 \\
1.774,7837 \\
1.755,0300 \\
1.703,9259 \\
1.555,0335\end{array}$ & $\begin{array}{r}3: 10^{-4} \\
37 \cdot 10^{-4} \\
33: 10^{-4} \\
44: 10^{-4} \\
52: 10^{-4} \\
97: 10^{-4} \\
14: 10^{-4} \\
83 \cdot 10^{-4} \\
129 \cdot 10^{-6} \\
105: 10^{-4} \\
140: 10^{-4} \\
9: 10^{-4} \\
57: 10^{-4} \\
56 \cdot 10^{-4} \\
53: 10^{-4}\end{array}$ \\
\hline \multicolumn{2}{|c|}{$\begin{array}{l}\text { Triangolazione VI } \\
4-11 / \mathrm{X} 1951\end{array}$} & \multicolumn{2}{|c|}{ Quota Bacino - Pieno } & & \\
\hline \multicolumn{2}{|c|}{$\begin{array}{l}\text { TRIANGOLAzIONE VII } \\
21-30 / 1111952\end{array}$} & \multicolumn{2}{|c|}{ Quota Bacino - 644,50 } & & \\
\hline
\end{tabular}

Tabella 7

\begin{tabular}{|c|c|c|c|c|c|}
\hline \multirow{2}{*}{ VERTICI } & \multicolumn{2}{|c|}{ Triangolazione VII } & \multicolumn{2}{|c|}{ Triangolazione VIII } & \multirow{2}{*}{$\begin{array}{c}T(V I I I-V I I) \\
\sqrt{\Delta^{2} x+\Delta^{2} y} \\
\mathrm{~mm}\end{array}$} \\
\hline & $\begin{array}{c}X(T \cdot V I I) \\
\mathrm{m}\end{array}$ & $\begin{array}{c}Y(T \cdot V I I) \\
\mathbf{m}\end{array}$ & $\begin{array}{c}X(T \cdot V I I I) \\
\mathbf{m}\end{array}$ & $\underset{\mathbf{m}}{Y(T \cdot V I I I)}$ & \\
\hline $13 \mathrm{M}$ & $1.000,0000$ & $1.000,0000$ & $1.000,0000$ & $1.000,0000$ & - \\
\hline $11 M$ & 784,3212 & $1.462,7050$ & 784,3212 & 1. 462,7048 & $10^{-4}$ \\
\hline 6 & $1.053,8507$ & $1.374,3435$ & $1.063,8465$ & 1. 374,3476 & $58 \cdot 10^{-4}$ \\
\hline 7 & 802,4220 & $1.593,3031$ & 802,4209 & 1.593.2997 & $36 \cdot 10^{-4}$ \\
\hline 8 & 1. 194,8978 & $1.828,7661$ & 1. 194,8867 & $1.828,7551$ & $156 \cdot 10^{-4}$ \\
\hline 9 & 760,4966 & 1. 799,6374 & 760,4939 & 1. 7996299 & $80 \cdot 10^{-4}$ \\
\hline 10 & 1. 114,9315 & $2.075,7840$ & 1. 114,9202 & $2.075,7701$ & $179 \cdot 10^{-4}$ \\
\hline 11 & 745,1631 & $2.008,5310$ & 745,1638 & $2.008,5210$ & $100 \cdot 10^{-4}$ \\
\hline 12 & 808,4699 & $2.389,4477$ & 808,4726 & $2.389,4231$ & $247 \cdot 10^{-4}$ \\
\hline 13 & 1. 353,8892 & $2.433,5562$ & 1. 353,8679 & $2.433,5267$ & $364 \cdot 10^{-4}$ \\
\hline 14 & 1. 144,2957 & 1. 791,6675 & 1. 144,2836 & 1. 791,6562 & $166 \cdot 10^{-4}$ \\
\hline 15 & 844,3736 & $1.791,4290$ & 844,3723 & 1. 791,4099 & $191 \cdot 10^{-1}$ \\
\hline 16 & $1.094,7848$ & 1. 774,7837 & $1.094,7754$ & 1. 774,7772 & $114 \cdot 10^{-4}$ \\
\hline 17 & 906,1547 & 1. 755,0300 & 906,1500 & 1. 755,0242 & $75 \cdot 10^{-4}$ \\
\hline 18 & 1. 136,3309 & 1.703,9259 & 1. 136,3246 & 1. 703,9229 & $70 \cdot 10^{-1}$ \\
\hline 19 & 1. 159,1596 & $1.555,0335$ & 1. 159,1530 & $1.555,0287$ & $82 \cdot 10^{-4}$ \\
\hline \multicolumn{2}{|c|}{$\begin{array}{l}\text { TRIANGOLAzione VII } \\
21-30 / 1111952\end{array}$} & \multicolumn{2}{|c|}{ Quota Bacino - 644,50 } & & \\
\hline \multicolumn{2}{|c|}{$\begin{array}{l}\text { Triangolazione VIII } \\
16-24 \text { /VI } 1952\end{array}$} & \multicolumn{2}{|c|}{ Quota Bacino - 683,50} & & \\
\hline
\end{tabular}


Tabella 8

\begin{tabular}{|c|c|c|c|c|c|c|c|c|c|c|c|}
\hline \multirow{3}{*}{$\begin{array}{c}\text { Vertici } \\
\text { Triangoli }\end{array}$} & \multicolumn{11}{|c|}{ TRIANGOLAZIONE I I } \\
\hline & $\frac{d u}{d x}$ & $\frac{d u}{d y}$ & $\frac{d v}{d x}$ & $\frac{d v}{d y}$ & $\Delta$ & $\sigma$ & $\omega$ & $\sigma_{\max }$ & $\gamma_{1}$ & $\gamma_{2}$ & $\theta$ \\
\hline & \multicolumn{10}{|c|}{$10^{-8}$} & \\
\hline$(10)(12)(13)$ & 305,6 & 483,5 & 41,1 & 512,0 & 817,6 & 262,3 & 221,2 & 563,7 & $1.381,3$ & 253,9 & $55^{\circ} 45^{\prime}$ \\
\hline$(11)(12)(13)$ & 323,4 & 263,9 & 45,1 & 462,4 & 785,8 & 154,5 & 109,4 & 338,8 & $1.124,6$ & 447,0 & $57^{\circ} 07^{\prime}$ \\
\hline$(10)(11)(12)$ & 116,1 & 298,4 & $-\quad 1,7$ & 470,2 & 586,3 & 148,4 & 150,1 & 461,9 & $1.048,2$ & 124,4 & $70^{\circ} 02^{\prime}$ \\
\hline$(8)(11)(10)$ & 201,9 & $-173,5$ & 11,0 & 400,3 & 602,2 & $-81,3$ & $-92,3$ & 256,4 & 858,6 & 345,8 & $-70^{\circ} 21^{\prime}$ \\
\hline (9) (11) (10) & 130,3 & 220,2 & 24,3 & 327,3 & 457,6 & 122,3 & 98,0 & 331,6 & 789,2 & 126,0 & $65^{\circ} 11^{\prime}$ \\
\hline$(8)(9)(10)$ & 389,7 & $-112,7$ & $-23,8$ & 389,0 & 778,7 & $-68,3$ & $-44,5$ & 136,5 & 915,2 & 642,2 & $-44^{\circ} 5 l^{\prime}$ \\
\hline (8) (9) (11) & 366,2 & 237,5 & $-19,4$ & 324,1 & 690,3 & 109,1 & 128,5 & 222,1 & 912,4 & 468,2 & $39^{\circ} 32^{\prime}$ \\
\hline$(7)(8)(9)$ & 378,6 & 52,7 & 0,03 & 33,9 & 412,5 & 26,4 & 26,4 & 348,7 & 761,2 & 63,8 & $4^{\circ} 21^{\prime}$ \\
\hline$(6)(9)(8)$ & 367,2 & 222,7 & $-12,4$ & 219,5 & 586,7 & 105,2 & 117,6 & 257,0 & 843,7 & 329,7 & $27^{\circ} 28^{\prime}$ \\
\hline$(6)(7)(8)$ & 255,9 & 257,3 & $-133,9$ & 257,2 & 513,1 & 61,7 & 195,6 & 123,4 & 636,5 & 389,7 & $45^{\circ} 18^{\prime}$ \\
\hline$(6)(7)(9)$ & 13,0 & $-21,6$ & $-399,0$ & $-47,2$ & $-34,2$ & $-210,3$ & 188,7 & 424,9 & 390,7 & $-459,1$ & $-40^{\circ} 56^{\prime}$ \\
\hline$(6)(7)(11 M)$ & 111,7 & 91,7 & 61,4 & 481,5 & 593,2 & 76,6 & 15,2 & 400,2 & 993,4 & 193,0 & $78^{\circ} 46^{\prime}$ \\
\hline$(6)(7)(13 M)$ & 75,8 & 50,5 & $-380,1$ & $-25,5$ & 50,3 & $-164,8$ & 215,3 & 344,8 & 395,1 & $-294,5$ & $-36^{\circ} 28^{\prime}$ \\
\hline$(6)(11 M)(13 M)$ & 97,2 & 47,5 & $-117,2$ & $-63,2$ & 33,9 & $-34,9$ & 82,4 & 175,0 & 208,9 & $-141,1$ & $-11^{\circ} 45^{\prime}$ \\
\hline$(10)(11)(13)$ & 51,6 & 653,2 & $-16,3$ & 550,3 & 601,9 & 318,5 & 334,8 & 808,9 & $1.410,8$ & $-207,0$ & $64^{\circ} 02^{\prime}$ \\
\hline
\end{tabular}


Tabella 9

\begin{tabular}{|c|c|c|c|c|c|c|c|c|c|c|c|}
\hline \multirow{3}{*}{$\begin{array}{c}\text { Vertici } \\
\text { Triangoli }\end{array}$} & \multicolumn{11}{|c|}{ TRIA N GOLAZION E I I I } \\
\hline & $\frac{d u}{d x}$ & $\frac{d u}{d y}$ & $\frac{d v}{d x}$ & $\frac{d v}{d y}$ & $\Delta$ & $\sigma$ & $\omega$ & $\sigma_{\max }$ & $\gamma_{1}$ & $\gamma_{2}$ & $\theta$ \\
\hline & \multicolumn{10}{|c|}{$10^{-8}$} & \\
\hline$(10)(12)(13)$ & $-667,7$ & $-244,4$ & $-107,2$ & $-602,0$ & $-1.269,7$ & $-175,8$ & $-68,6$ & 357,7 & $-\quad 912,0$ & $-1.627,4$ & $-50^{\circ} 18^{\prime}$ \\
\hline$(11)(12)(13)$ & $-677,1$ & $-129,0$ & $-123,3$ & $-402,1$ & $-1.079,2$ & $-126,1$ & $-2,8$ & 373,2 & $-706,0$ & $-1.452,4$ & $-68^{\circ} 38^{\prime}$ \\
\hline$(10)(11)(12)$ & $-568,2$ & $-147,1$ & 65,3 & $-433,5$ & $-1.001,7$ & $-40,9$ & $-106,2$ & 157,6 & $-844,1$ & $-1.159,3$ & $-74^{\circ} 22^{\prime}$ \\
\hline$(10)(11)(13)$ & $-534,3$ & $-333,5$ & 124,1 & $-756,4$ & $-1.290,7$ & $-104,7$ & $-228,8$ & 305,3 & $-985,5$ & $-1.595,9$ & $-21^{\circ} 39^{\prime}$ \\
\hline$(8)(11)(10)$ & $-587,6$ & $-40,4$ & 80,4 & $-516,4$ & $-1 \cdot 140,0$ & 20,0 & $-60,4$ & 81,7 & $-1.022,3$ & $-1.185,7$ & $75^{\circ} 20^{\prime}$ \\
\hline$(9)(11)(10)$ & $-568,2$ & $-147,0$ & 40,8 & $-298,6$ & $-\quad 866,8$ & $-53,1$ & $-93,9$ & 289,8 & $-\quad 577,0$ & $-1.156,6$ & $-79^{\circ} 15^{\prime}$ \\
\hline (8) (9) $(10)$ & $-638,4$ & $-56,9$ & 187,9 & $-428,8$ & $-1.121,1$ & 65,5 & $-122,4$ & 203,4 & $-\quad 917,7$ & . $1.324,6$ & $69^{\circ} 57^{\prime}$ \\
\hline (8) (9) (11) & $-632,0$ & $-151,7$ & 171,3 & $-289,0$ & $-\quad 921,0$ & 9,8 & $-161,5$ & 343,6 & $-\quad 577,4$ & $-1.264,6$ & $88^{\circ} 22^{\prime}$ \\
\hline (7) (8) (9) & $-638,4$ & $-57,0$ & 180,3 & $-423,7$ & $-1.062,1$ & 61,6 & $-118,6$ & 247,6 & $-814,5$ & $-1.309,7$ & $75^{\circ} 04^{\prime}$ \\
\hline (6) (9) (8) & $-623,9$ & $-272,8$ & 170,7 & $-279,6$ & $-\quad 903,5$ & $-51,0$ & $-221,7$ & 359,1 & - $\quad 544,4$ & $-1.262,6$ & $-81^{\circ} 44^{\prime}$ \\
\hline$(6)(7)(8)$ & $-482,6$ & $-316,7$ & 76,3 & $-250,3$ & $-\quad 732,9$ & $-120,2$ & $-196,5$ & 334,3 & $-\quad 398,6$ & $-1.067,2$ & $-67 \circ 00^{\prime}$ \\
\hline$(6)(7)(9)$ & $-174,4$ & 37,3 & $-129,6$ & $-486,7$ & $-\quad 661,1$ & $-46,1$ & 83,4 & 325,7 & $-\quad 335,4$ & $-\quad 986,8$ & $-8^{\circ} 16^{\prime}$ \\
\hline$(6)(7)(11 M)$ & $-291,6$ & $-97,4$ & $-34,9$ & $-378,0$ & $-\quad 669,6$ & $-66,2$ & $-31,3$ & 158,0 & $-\quad 511,6$ & $-\quad 827,6$ & $-28^{\circ} 25^{\prime}$ \\
\hline (6) (7) (13M) & $-326,4$ & $-137,4$ & 305,0 & 12,2 & $-\quad 314,2$ & 82,8 & $-221,2$ & 377,8 & 63,6 & $-\quad 692,0$ & $76^{\circ} 50^{\prime}$ \\
\hline$(6)(11 M)(13 M)$ & $-305,7$ & $-140,3$ & 102,6 & 41,3 & $-\quad 264,4$ & $-18,8$ & $-121,5$ & 349,0 & 84,6 & $-613,4$ & $-86^{\circ} 54^{\prime}$ \\
\hline
\end{tabular}


Tabella 10

\begin{tabular}{|c|c|c|c|c|c|c|c|c|c|c|c|}
\hline \multirow{3}{*}{$\begin{array}{c}\text { Vertici } \\
\text { Triangoli }\end{array}$} & \multicolumn{11}{|c|}{ TRIANGOLAZIONE I V } \\
\hline & $\frac{d u}{d x}$ & $\frac{d u}{d y}$ & $\frac{d v}{d x}$ & $\frac{d v}{d y}$ & $\Delta$ & $\sigma$ & $\omega$ & $\sigma_{\max }$ & $\gamma_{1}$ & $\gamma_{2}$ & 0 \\
\hline & \multicolumn{10}{|c|}{$10^{-8}$} & \\
\hline$(10)(12)(13)$ & 597,6 & 137,5 & 60,3 & 502,1 & $1.099,7$ & 98,9 & 38,6 & 219,6 & 1. 319,3 & 880,1 & $32^{\circ} 06^{\prime}$ \\
\hline$(11)(12)(13)$ & 612,7 & $-49,3$ & 74,2 & 329,0 & 941,7 & 12,3 & $-61,7$ & 284,8 & 1. 226,5 & 656,9 & $2032^{\prime}$ \\
\hline$(10)(11)(12)$ & 436,4 & $-20,0$ & $-89,1$ & 356,1 & 792,5 & $-54,5$ & 34,5 & 135,5 & 928,0 & 657,0 & $-26^{\circ} 51^{\prime}$ \\
\hline$(10)(11)(13)$ & 381,5 & 281,9 & $-140,0$ & 635,8 & $1.017,3$ & 70,9 & 210,9 & 291,2 & $1.308,5$ & 726,1 & $75^{\circ} 25^{\prime}$ \\
\hline$(8)(11)(10)$ & 408,0 & 136,1 & $-107,8$ & 459,0 & 867,0 & 14,1 & 121,9 & 58,3 & 925,3 & 808,7 & $75^{\circ} 29^{\prime}$ \\
\hline$(9)(11)(10)$ & 392,7 & 220,3 & $-79,9$ & 305,3 & 698,0 & 70,2 & 150,1 & 136,2 & 834,2 & 561,8 & $19^{\circ} 10^{\prime}$ \\
\hline$(8)(9)(10)$ & 448,1 & 149,1 & $-181,1$ & 435,2 & 883,3 & $-16,0$ & 165,1 & 34,5 & 917,8 & 848,8 & $34^{\circ} 02^{\prime}$ \\
\hline$(8)(9)(11)$ & 443,1 & 224,0 & $-172,0$ & 298,6 & 741,7 & 26,0 & 198,0 & 153,6 & 895,3 & 588,1 & $9^{\circ} 56^{\prime}$ \\
\hline$(7)(8)(9)$ & 449,4 & 130,1 & $-199,6$ & 710,7 & $1,160,1$ & $-34,7$ & 164,8 & 270,4 & $1.430,5$ & 889,5 & $-82^{\circ} 33^{\prime}$ \\
\hline$(6)(9)(8)$ & 450,4 & 115,5 & $-178,4$ & 394,3 & 844,7 & $-31,4$ & 146,9 & 84,3 & 929,0 & 760,4 & $24^{\circ} 09^{\prime}$ \\
\hline$(6)(7)(8)$ & 459,9 & 112,5 & 28,8 & 330,0 & 789,9 & 70,6 & 41,8 & 191,9 & 981,8 & 598,0 & $23^{\circ} 41^{\prime}$ \\
\hline (6) (7) (9) & 480,7 & 136,4 & 480,7 & 848,8 & $1.329,5$ & 308,6 & $-172,1$ & 718,6 & $2.048,1$ & 610,9 & $60^{\circ} 24^{\prime}$ \\
\hline$(6)(7)(11 M)$ & 346,8 & $-17,4$ & 7,3 & 305,3 & 652,1 & $-5,1$ & $-12,4$ & 42,7 & 694,8 & 609,4 & $-6^{\circ} 46^{\prime}$ \\
\hline$(6)(7)(13 M)$ & 474,1 & 172,1 & $-258,7$ & $-\quad 0,2$ & 473,9 & $-43,3$ & 215,4 & 482,1 & 956,0 & $-8,2$ & $-5^{\circ} 02^{\prime}$ \\
\hline$(6)(11 M)(13 M)$ & 413,5 & 186,3 & $-100,3$ & $-23,0$ & 390,5 & 43,0 & 143,3 & 444,9 & 835,4 & $-54,4$ & $5^{0} 35^{\prime}$ \\
\hline
\end{tabular}


Tabella 11

\begin{tabular}{|c|c|c|c|c|c|c|c|c|c|c|c|}
\hline \multirow{3}{*}{$\begin{array}{c}\text { Vertici } \\
\text { Triangoli }\end{array}$} & \multicolumn{11}{|c|}{ TRIA NGOLAZIONE V } \\
\hline & $\frac{d u}{d x}$ & $\frac{d u}{d y}$ & $\frac{d v}{d x}$ & $\frac{d v}{d y}$ & $\Delta$ & $\sigma$ & $\omega$ & $\sigma_{m a x}$ & $\gamma_{1}$ & $\gamma_{2}$ & $\theta$ \\
\hline & \multicolumn{10}{|c|}{$10^{-8}$} & \\
\hline$(10)(12)(13)$ & $-503,5$ & 14,9 & $-17,8$ & $-460,5$ & $-964,0$ & $-1,5$ & $-16,4$ & 43,1 & $-920,9$ & $-1.007,1$ & $-88^{\circ} 04^{\prime}$ \\
\hline$(11)(12)(13)$ & $-114,6$ & 15,2 & $-29,7$ & $-312,7$ & $-\quad 827,3$ & † 60,7 & 90,4 & 235,6 & $-591,7$ & $-1.062,9$ & $74^{\circ} 29^{\prime}$ \\
\hline$(10)(11)(12)$ & $-386,0$ & 129,8 & $-109,8$ & $-335,9$ & $-\quad 721,9$ & $+119,8$ & 10,0 & 244,8 & $-477,1$ & $-\quad 966,7$ & $48^{\circ} 19^{\prime}$ \\
\hline$(10)(11)(13)$ & $-340,9$ & $-90,4$ & 153,2 & $-574,7$ & $-920,6$ & $+31,4$ & $-121,8$ & 237,3 & $-683,3$ & $-1.157,9$ & $7^{\circ} 53^{\prime}$ \\
\hline$(8)(11)(10)$ & $-331,8$ & $-168,1$ & 132,2 & $-459,2$ & $-\quad 791,0$ & $-17,9$ & $-150,1$ & 132,3 & $-658,7$ & $-923,3$ & $-7^{\circ} 46^{\prime}$ \\
\hline$(9)(11)(10)$ & $-340,4$ & $-120,7$ & 97,0 & $-265,7$ & $-606,1$ & $-11,8$ & $-108,8$ & 784,0 & $-527,7$ & $-684,5$ & $-81^{\circ} 12^{\prime}$ \\
\hline$(8)(9)(10)$ & $-309,2$ & $-160,8$ & 224,4 & $-429,3$ & $-\quad 738,5$ & $+33,8$ & $-192,6$ & 135,9 & $-602,6$ & - 874,4 & $13^{\circ} 57^{\prime}$ \\
\hline (8) (9) (11) & $-312,0$ & $-118,6$ & 212,9 & $-257,2$ & $-\quad 569,2$ & $+47,1$ & $-165,7$ & 109,1 & $-460,1$ & $-\quad 678,3$ & $60^{\circ} 05^{\prime}$ \\
\hline$(10)(14)(15)$ & $-353,4$ & $-71,7$ & 253,6 & $-234,2$ & $-\quad 587,6$ & $+90,9$ & $-162,6$ & 217,5 & $-370,1$ & $-805,1$ & $61^{\circ} 37^{\prime}$ \\
\hline$(11)(14)(15)$ & $-353,4$ & $-78,6$ & 253,4 & 42,1 & $-\quad 311,3$ & $+87,4$ & $-166,0$ & 432,4 & 121,1 & $-\quad 743,7$ & $78^{\circ} 05^{\prime}$ \\
\hline$(7)(8)(9)$ & $-380,6$ & $-169,3$ & 288,9 & $-496,3$ & $-\quad 804,9$ & $+29,8$ & $-199,1$ & 196,9 & $-608,0$ & $-1.001,8$ & $8^{\circ} 47^{\prime}$ \\
\hline (7) (9) (18) & 16,3 & $-103,3$ & 101,2 & $-522,3$ & $-\quad 506,0$ & $-0,1$ & $-102,2$ & 522,3 & 16,3 & $-1.028,3$ & $82^{\circ} 40^{\prime}$ \\
\hline (6) (9) (8) & $-316,2$ & $-55,9$ & 218,8 & $-345,2$ & $-\quad 661,4$ & $+81,4$ & $-137,3$ & 165,5 & $-495,9$ & $-\quad 826,9$ & $39^{\circ} 58^{\prime}$ \\
\hline$(6)(17)(16)$ & $-466,6$ & $-99,6$ & 101,5 & $-412,4$ & $-\quad 879,0$ & †. 1,0 & $-100,5$ & 54,2 & $-824,8$ & $-\quad 933,2$ & $-11^{\circ} 30^{\prime}$ \\
\hline$(7)(18)(19)$ & $-88,2$ & $-701,4$ & 67,1 & $-419,4$ & $-\quad 507,7$ & $-317,1$ & $-384,2$ & 458,4 & $-49,3$ & $-966,1$ & $89^{\circ} 00^{\prime}$ \\
\hline$(6)(7)(8)$ & $-390,5$ & $-32,8$ & 119,9 & $-314,5$ & $-705,0$ & $+43,6$ & $-76,3$ & 115,6 & $-589,4$ & $-820,6$ & $65^{\circ} 33^{\prime}$ \\
\hline$(6)(7)(9)$ & $-552,5$ & $-218,9$ & $-95,9$ & $-56,2$ & $-1.114,7$ & $-157,4$ & $-61,5$ & 314,9 & $-799,8$ & $-1.429,6$ & $-44^{\circ} 07^{\prime}$ \\
\hline (6) (7) (11M) & $-400,3$ & $-44,1$ & 149,0 & $-281,0$ & $-681,3$ & $+52,5$ & $-96,6$ & 158,9 & $-522,4$ & $-840,2$ & $69^{\circ} 21^{\prime}$ \\
\hline$(6)(7)(13 M)$ & $-534,6$ & $-198,2$ & 478,1 & 96,8 & $-\quad 437,8$ & $+139,9$ & $-338,2$ & 690,7 & 252,9 & $-1.128,5$ & $78^{\circ} 03^{\prime}$ \\
\hline$(6)(11 M)(13 M)$ & $-454,6$ & $-209,7$ & 282,1 & 125,0 & $-\quad 329,6$ & $+36,2$ & $-245,9$ & 584,1 & $-254,5$ & $-\quad 913,7$ & $84^{\circ} 10^{\prime}$ \\
\hline
\end{tabular}


Tabella 12

\begin{tabular}{|c|c|c|c|c|c|c|c|c|c|c|c|}
\hline \multirow{3}{*}{$\begin{array}{c}\text { Vertici } \\
\text { Triangoli }\end{array}$} & \multicolumn{11}{|c|}{ TRIANGOLAZIONEVI } \\
\hline & $\frac{d u}{d x}$ & $\frac{d u}{d y}$ & $\frac{d v}{d x}$ & $\frac{d v}{d y}$ & $\Delta$ & $\sigma$ & $\omega$ & $\sigma_{\max }$ & $\gamma_{1}$ & $\gamma_{2}$ & $\theta$ \\
\hline & \multicolumn{10}{|c|}{$10^{-8}$} & \\
\hline$(10)(12)(13)$ & 369,6 & 214,4 & $-29,6$ & 388,8 & 758,4 & 92,4 & 122,0 & 185,8 & 944,2 & 572,6 & $47^{\circ} 58^{\prime}$ \\
\hline$(11)(12)(13)$ & 387,2 & $-\quad 4,0$ & $-14,7$ & 204,6 & 591,8 & $-9,3$ & 5,3 & 183,6 & 775,4 & 408,2 & $-3^{\circ} 04^{\prime}$ \\
\hline$(10)(11)(12)$ & 181,1 & 30,3 & $-185,5$ & 233,5 & 414,6 & $-79,1$ & 109,4 & 166,7 & 581,3 & 247,9 & $-54^{\circ} 10^{\prime}$ \\
\hline$(10)(11)(13)$ & 116,9 & 383,1 & $-242,6$ & 531,0 & 647,9 & 70,2 & 312,8 & 437,3 & $1.085,2$ & 210,6 & $80^{\circ} 38^{\prime}$ \\
\hline$(8)(11)(10)$ & 164,4 & 122,0 & $-136,5$ & $-52,3$ & 112,1 & $-7,2$ & 129,2 & 217,2 & 329,3 & $-105,1$ & $-1^{\circ} 59^{\prime}$ \\
\hline$(9)(11)(10)$ & 197,0 & $-57,3$ & $-163,9$ & 98,1 & 295,1 & $-110,6$ & 53,2 & 242,3 & 537,4 & 52,8 & $-32^{\circ} 57^{\prime}$ \\
\hline$(8)(9)(10)$ & 78,8 & 94,3 & $-64,8$ & $-29,1$ & 49,7 & 14,7 & 79,5 & 111,9 & 161,6 & $-111,9$ & $7^{\circ} 43^{\prime}$ \\
\hline$(8)(9)(11)$ & 89,6 & $-65,2$ & $-73,8$ & 104,7 & 194,3 & $-69,5$ & 4,3 & 139,8 & 334,1 & 54,5 & $-48^{\circ} 06^{\prime}$ \\
\hline$(10)(14)(15)$ & 139,9 & 134,1 & $-70,1$ & 87,8 & 227,7 & 32,0 & 102,1 & 82,5 & 310,2 & 145,2 & $25^{\circ} 25^{\prime}$ \\
\hline$(11)(14)(15)$ & 140,0 & 96,2 & -702 & 244,3 & 384,3 & 13,0 & 83,2 & 107,5 & 491,8 & 276,8 & $83^{\circ} 00^{\prime}$ \\
\hline$(7)(8)(9)$ & 82,4 & 41,0 & $-71,0$ & 63,1 & 145,5 & $-15,0$ & 56,0 & 35,7 & 181,2 & 109,8 & $-28^{\circ} 40^{\prime}$ \\
\hline$(7)(9)(18)$ & $-74,9$ & 9,0 & $-122,3$ & 52,7 & $-22,2$ & $-60,7$ & 65,6 & 124,9 & 102,7 & $-147,1$ & $-65^{\circ} 50^{\prime}$ \\
\hline$(6)(9)(8)$ & 85,3 & $-\quad 2,3$ & $-75,7$ & 133,5 & 218,8 & $-39,0$ & 36,7 & 91,7 & 310,5 & 127,1 & $-60^{\circ} 5 l^{\prime}$ \\
\hline$(6)(17)(16)$ & 239,8 & $-62,0$ & 41,6 & 210,5 & 450,3 & $-10,2$ & $-51,8$ & 35,7 & 486,0 & 414,6 & $-17025^{\prime}$ \\
\hline$(7)(18)(19)$ & $-129,8$ & 174,9 & $-36,2$ & $-207,0$ & $-336,8$ & 69,3 & 105,5 & 158,7 & $-178,1$ & $-495,5$ & $30^{\circ} 26^{\prime}$ \\
\hline$(6)(7)(8)$ & 113,7 & $-11,1$ & $-121,8$ & 147,8 & 261,5 & $-66,4$ & 55,3 & 137,2 & 398,7 & 124,3 & $-52^{\circ} 12^{\prime}$ \\
\hline$(6)(7)(9)$ & 175,4 & 59,9 & $-222,4$ & 32,4 & 207,8 & $-81,2$ & 141,1 & 216,5 & 424,3 & $-8,7$ & $-24^{\circ} 20^{\prime}$ \\
\hline$(6)(7)(11 M)$ & 205,2 & 94,1 & $-217,6$ & 37,8 & 243,0 & $-61,8$ & 155,9 & 208,0 & 451,0 & 35,0 & $-18^{\circ} 12^{\prime}$ \\
\hline$(6)(7)(13 M)$ & 210,9 & 100,6 & $-365,8$ & $-131,8$ & 79,1 & $-132,3$ & 232,9 & 433,0 & 512,1 & 353,9 & $-70^{\circ} 46^{\prime}$ \\
\hline (6) $(1 \mathrm{M})(13 \mathrm{M})$ & 207,5 & 101,0 & $-277,4$ & $-144,4$ & 63,1 & $-88,2$ & 189,2 & 393,6 & 456,7 & $-330,5$ & $-13^{\circ} 18^{\prime}$ \\
\hline
\end{tabular}


Tabella 13

\begin{tabular}{|c|c|c|c|c|c|c|c|c|c|c|c|}
\hline \multirow{3}{*}{$\begin{array}{c}\text { Vertici } \\
\text { Triangoli }\end{array}$} & \multicolumn{11}{|c|}{ TRIANGOIAZIONE VII } \\
\hline & $\frac{d u}{d x}$ & $\frac{d u}{d y}$ & $\frac{d v}{d x}$ & $\frac{d v}{d y}$ & $\Delta$ & $\sigma$ & $\omega$ & $\sigma_{\max }$ & $\gamma_{1}$ & $\gamma_{2}$ & 0 \\
\hline & \multicolumn{10}{|c|}{$10^{-8}$} & \\
\hline$(10)(12)(13)$ & 9,2 & $-239,5$ & 90,7 & $-10,2$ & $-\quad 7,3$ & $-74,4$ & $-165,1$ & 149,4 & 142,1 & $-\quad 156,7$ & $-42 \circ 30^{\prime}$ \\
\hline$(11)(12)(13)$ & $-5,1$ & $-140,9$ & 80,5 & 115,3 & 110,2 & $-30,2$ & $-110,7$ & 134,7 & 244,9 & 24,5 & $-76 \circ 40^{\prime}$ \\
\hline$(10)(11)(12)$ & 87,9 & $-156,4$ & 199,0 & 95,6 & 183,5 & 21,3 & $-177,7$ & 43,3 & 226,8 & 140,2 & $50 \circ 08^{\prime}$ \\
\hline$(10)(11)(13)$ & 116,9 & $-315,7$ & 235,9 & $-107,2$ & 9,7 & $-39,9$ & $-275,8$ & 237,9 & 247,6 & $-\quad 228,2$ & $-9048^{\prime}$ \\
\hline$(8)(11)(10)$ & 54,1 & 29,7 & 166,8 & 272,6 & 326,7 & 98,2 & $-68,5$ & 293,9 & 620,5 & 32,9 & $69 \circ 01^{\prime}$ \\
\hline$(9)(11)(10)$ & 70,7 & $-61,8$ & 242,7 & $-144,9$ & $-74,2$ & 90,4 & $-152,2$ & 281,4 & 207,2 & $-\quad 355,6$ & $19059^{\prime}$ \\
\hline$(8)(9)(10)$ & 10,5 & 15,5 & $-32,4$ & 208,1 & 218,6 & $-8,4$ & 23,9 & 198,3 & 416,9 & 20,3 & $-87 \circ 33^{\prime}$ \\
\hline$(8)(9)(11)$ & 15,9 & 65,9 & $-7,5$ & $-163,3$ & $-147,4$ & $-36,7$ & $-29,2$ & 193,6 & 46,3 & $-341,1$ & $-11010^{\prime}$ \\
\hline$(10)(14)(15)$ & $-200,2$ & $-144,7$ & $-203,4$ & 31,8 & 232,0 & $-174,1$ & 29,3 & 386,7 & 618,7 & $-154,7$ & $-32 \circ 06^{\prime}$ \\
\hline$(11)(14)(15)$ & 200,0 & 49,9 & $-202,8$ & $-673,0$ & $-473,0$ & $-76,4$ & 126,3 & 886,3 & 413,3 & $-1.359,3$ & $-4058^{\prime}$ \\
\hline$(7)(8)(9)$ & 7,2 & 64,5 & $-23,0$ & 68,0 & 75,2 & 20,7 & 43,7 & 73,6 & 148,8 & 1,6 & $72 \circ 50^{\prime}$ \\
\hline (7) $(9)(18)$ & 73,0 & 77,8 & 8,3 & 74,4 & 147,4 & 43,1 & 34,7 & 113,8 & 261,2 & 33,6 & $53 \circ 14^{\prime}$ \\
\hline$(6)(9)(8)$ & 6,8 & 70,5 & $-23,5$ & 75,5 & 82,3 & 23,5 & 47,0 & 83,2 & 165,5 & 0,9 & $72 \circ 48^{\prime}$ \\
\hline$(6)(17)(16)$ & $-268,4$ & 82,4 & $-12,6$ & $-31,2$ & $-299,6$ & 34,9 & 47,5 & 247,3 & $-52,3$ & $-\quad 546,9$ & $81043^{\prime}$ \\
\hline$(7)(18)(19)$ & 115,2 & $-49,5$ & $-0,4$ & 100,7 & 215,9 & $-24,9$ & $-24,5$ & 51,9 & 267,8 & 164,0 & $-3605 l^{\prime}$ \\
\hline$(6)(7)(8)$ & 2,8 & 71,7 & $-28,4$ & 77,0 & 79,8 & 21,6 & 50,1 & 85,9 & 165,7 & 6,1 & $74^{\circ} 52^{\prime}$ \\
\hline$(6)(7)(9)$ & $-5,8$ & 61,8 & $-39,1$ & 64,8 & 59,0 & 11,3 & 50,4 & 74,2 & 133,2 & 15,2 & $81006^{\prime}$ \\
\hline$(6)(7)(11 \mathrm{M})$ & $-41,3$ & 21,0 & 93,3 & 230,4 & 189,1 & 57,2 & $-36,2$ & 294,8 & 483,9 & $-\quad 105,7$ & $78 \circ 35^{\prime}$ \\
\hline (6) (7) (13M) & $-82,0$ & $-25,6$ & $-66,2$ & 33,6 & $-48,4$ & $-45,9$ & 20,3 & 147,6 & 99,2 & $-196,0$ & $-70046^{\prime}$ \\
\hline$(6)(11 M)(13 \mathrm{M})$ & $-57,8$ & $-29,1$ & 28,8 & 19,9 & $-37,9$ & $-\quad 0,2$ & $-28,9$ & 77,7 & 39,8 & $-\quad 115,6$ & $-0053^{\prime}$ \\
\hline
\end{tabular}


Tabella 14

\begin{tabular}{|c|c|c|c|c|c|c|c|c|c|c|c|}
\hline \multirow{3}{*}{$\begin{array}{l}\text { Vertici } \\
\text { Triangoli }\end{array}$} & \multicolumn{11}{|c|}{ TRIA N G OLAZION E V I I I } \\
\hline & $\frac{d u}{d x}$ & $\frac{d u}{d y}$ & $\frac{d v}{d x}$ & $\frac{d v}{d y}$ & $\Delta$ & $\sigma$ & $\omega$ & $\sigma_{\max }$ & $\gamma_{1}$ & $\gamma_{2}$ & $\theta$ \\
\hline & \multicolumn{10}{|c|}{$10^{-8}$} & \\
\hline$(10)(12)(13)$ & $-441,2$ & 15,2 & $-57,7$ & $-397,5$ & $-838,7$ & $-21,3$ & 36,5 & 61,0 & $-\quad 777,7$ & $-899,7$ & $-67^{\circ} 54^{\prime}$ \\
\hline$(11)(12)(13)$ & $-450,3$ & 127,3 & $-59,6$ & $-373,4$ & $-823,7$ & 33,8 & 93,4 & 102,5 & $-721,2$ & $-926,2$ & $69^{\circ} 20^{\prime}$ \\
\hline$(10)(11)(12)$ & $-344,5$ & 109,8 & $-36,9$ & $-377,1$ & $-721,6$ & 36,4 & 73,3 & 79,9 & $-641,7$ & $-801,5$ & $32^{\circ} 59^{\prime}$ \\
\hline$(8)(11)(10)$ & $-305,1$ & $-106,9$ & $-79,4$ & $-143,1$ & $-448,2$ & 93,1 & $-13,7$ & 246,9 & $-\quad 201,3$ & $-695,1$ & $65^{\circ} 30^{\prime}$ \\
\hline$(9)(11)(10)$ & $-349,5$ & 137,1 & $-82,6$ & $-125,7$ & $-475,2$ & 27,2 & 109,8 & 230,3 & $-\quad 244,9$ & $-705,5$ & $83^{\circ} 09^{\prime}$ \\
\hline$(8)(9)(10)$ & $-188,7$ & $-69,2$ & $-71,2$ & $-140,4$ & $-329,1$ & $-70,2$ & 1,0 & 148,5 & $-\quad 180,6$ & $-477,6$ & $-54^{\circ} 30^{\prime}$ \\
\hline$(8)(9)(11)$ & $-203,3$ & 147,8 & $-72,2$ & $-125,0$ & $-328,3$ & 37,8 & 110,0 & 108,8 & $-\quad 219,5$ & $-437,1$ & $68^{\circ} 00^{\prime}$ \\
\hline$(7)(8)(9)$ & $-185,6$ & $-115,3$ & $-66,3$ & $-212,2$ & $-397,8$ & $-90,8$ & $-24,5$ & 183,5 & $-\quad 214,3$ & $-581,3$ & $-40^{\circ} 50^{\prime}$ \\
\hline (6) (9) (8) & $-187,1$ & $-93,8$ & $-59,5$ & $-313,8$ & $-500,9$ & $-76,6$ & $-17,1$ & 198,9 & $-\quad 302,0$ & $-699,8$ & $-25^{\circ} 13^{\prime}$ \\
\hline (6) (7) (8) & $-201,1$ & $-89,4$ & 7,0 & $-334,5$ & $-535,6$ & $-41,2$ & $-48,2$ & 156,8 & $-\quad 378,8$ & $-692,4$ & $-15^{\circ} 51^{\prime}$ \\
\hline$(6)(7)(9)$ & $-231,8$ & $-124,7$ & 152,2 & $-167,8$ & $-399,6$ & 13,7 & $-138,4$ & 69,7 & $-\quad 329,9$ & $-469,3$ & $78^{\circ} 23^{\prime}$ \\
\hline$(6)(7)(11 M)$ & $-175,5$ & $-59,9$ & 75,8 & $-255,5$ & $-431,0$ & 8,0 & $-67,9$ & 81,6 & $-\quad 349,4$ & $-512,6$ & $5^{\circ} 45^{\prime}$ \\
\hline$(6)(7)(13 M)$ & $-196,4$ & $-83,9$ & 349,8 & 59,2 & $-137,2$ & 132,9 & $-216,8$ & 368,8 & 231,6 & $-506,0$ & $66^{\circ} 56^{\prime}$ \\
\hline$(6)(11 M)(13 M)$ & $-183,9$ & $-85,7$ & 186,6 & 82,7 & $-101,2$ & 50,5 & $-136,1$ & 285,0 & 183,8 & $-386,2$ & $79^{\circ} 38^{\prime}$ \\
\hline$(10)(11)(13)$ & $-311,5$ & $-71,4$ & $-29,8$ & $-416,1$ & $-727,6$ & $-50,6$ & $-20,8$ & 145,5 & $-\quad 582,1$ & $-873,1$ & $-22^{\circ} 01^{\prime}$ \\
\hline$(7)(18)(19)$ & $-154,6$ & $-3,5$ & $-26,7$ & 116,8 & $-37,8$ & $-15,1$ & $-11,6$ & 273,1 & 235,3 & $-310,9$ & $-86^{\circ} 50^{\prime}$ \\
\hline$(6)(17)(16)$ & $-238,1$ & $-105,5$ & $-9,5$ & $-263,7$ & $-501,8$ & $-57,5$ & $-48,0$ & 117,8 & $-384,0$ & $-619,6$ & $-38^{\circ} 43^{\prime}$ \\
\hline$(7)(9)(18)$ & $-121,8$ & $-102,3$ & $-72,9$ & $-183,9$ & $-305,7$ & $-14,7$ & $-87,6$ & 186,2 & $-\quad 119,5$ & $-491,9$ & $-76^{\circ} 40^{\prime}$ \\
\hline$(10)(14)(15)$ & $-360,1$ & $-9,1$ & 260,1 & $-64,6$ & $-424,7$ & 125,5 & $-134,6$ & 387,7 & 37,0 & $-812,4$ & $69^{\circ} 50^{\prime}$ \\
\hline$(11)(14)(15)$ & $-360,0$ & $-72,4$ & 259,6 & 537,8 & 177,8 & 93,6 & $-166,0$ & 917,1 & $1.094,9$ & $-739,3$ & $84^{\circ} 07^{\prime}$ \\
\hline
\end{tabular}




\section{PARTE SECONDA}

Come è stato detto il lavoro di interpretazione geofisica, di cui qui si riassumono i risultati, è stato eseguito sui valori degli spostamenti della predisposta catena trigonometrica, osservati durante otto triangolazioni, compiute nel bacino di Pieve dall'Agosto 1949 al Giugno 1952.

La Tavola A riporta in scala detti spostamenti, secondo un ordine che rispetta quello con cui sono state eseguite le triangolazioni.

Le Tabelle da 1 a 7 riportano $i$ valori degli elementi della deformazione elastica relativi alle variazioni degli spostamenti osservate passando dalla prima alla seconda triangolazione, dalla seconda alla terza etc., ognuna per quindici terne di vertici.

Tali valori sono stati poi portati in pratica ed interpolati, cosi da poter costruire le tavole corrispondenti ad ognuno degli elementi della deformazione (Tabelle 1-7).

Le variazioni degli elementi della deformazione elastica conseguenti al passaggio dalla prima alla seconda triangolazione, dalla seconda alla terza, ecc. sono contenuti nelle sette tabelle da 8 a 14 .

\section{DLhatazione.}

La tav. Ia) dà la Dilatazione. Dobbiamo qui intercalare un'osservazione di carattere generale. La presenza della Diga viene praticamente a suddividere il bacino in due parti distinte, così da non consentire una continuità nell'andamento delle variazioni degli elementi in esame. Eे questo il motivo che $\mathrm{mi}$ ha indotto a dividere ogni tavola in due parti con soluzione di continuità da una all'altra.

A monte della diga le variazioni della dilatazione sono tutte positive e tendono a crescere procedendo in sponda sinistra. A valle della diga le variazioni sono pure generalmente positive fatta eccezione di una zona intorno al baricentro del Triangolo [6] [7] [9], in cui la variazione risulta negativa. Da questa zona però la variazione della dilatazione si fa positiva in tutte le direzioni con tendenza ad attenuarsi procedendo verso la base della triangolazione.
In altri termini dalla prima alla seconda triangolazione, per effetto degli spostamenti osservati e della loro direzione che in qualche modo li fa irraggiare, con riferimento al punto 7 nel quadrante di NE da Nord verso Est, si verifica nel terreno uno stiramento sia a monte che a valle della diga ad eccezione della zona di cui si è già detto, nella quale naturalmente si verifica una contrazione.

\section{Distorsione.}

L'andamento della distorsione è stato rappresentato graficamente nella tav. II $a$ ).

La dissimetria osservata negli spostamenti (per quanto si riferisce all'entità dello spostamento e alla sua direzione) si riflette in modo più chiaro nella distorsione, la quale nel gioco delle variazioni di queste due entità, ora è positiva ed ora negativa come risulta dalla tavola su accennata.

\section{Rotazione.}

L'andamento della rotazione è rappresentato dal grafico riportato nella tav. III $a$ ).

Come è detto nel testo la rotazione si esplica sotto forma di variazioni angolari intorno ad un asse normale al piano nel quale si osservano gli spostamenti, nel punto di osservazione.

Per quanto riguarda il segno - considerato positivo nel verso che va da Nord a Sud passando per Est - si osserva una certa analogia con il segno della dilatazione; analogia soltanto approssimativa naturalmente data la diversa natura fisica delle due grandezze in questione. Così nella tav. I c) si nota, per il segno, un andamento simile a quello della dilatazione salvo per quanto accade intorno al baricentro del triangolo [8] [11] [10].

\section{DEFORMAZIONI PRINCIPALI E LORO DIREZIONI}

La tav. V a) da la rappresentazione degli assi delle ellissi di deformazione, in corrispondenza del baricentro di ogni triangolo considerato.

I segmenti a tratto pieno corrispondono ad allungamenti, quelli tratteggiati a con- 
trazioni. L'unità di misura - come è stato detto - è $10^{-8} \mathrm{~cm}$.

Si nota subito che gli assi maggiori, a monte della Diga, hanno un orientamento verso la diga stessa e, in particolare, verso il punto di massima profondità del bacino. Nei pressi della diga, la presenza di quest'ultima altera alquanto l'andamento delle deformazioni che vengono, in qualche modo, influenzate dalle zone di spalla, alle quali le misure si riferiscono.

Questo vale pure per le stazioni immediatamente a valle della diga.

Le osservazioni più a valle, portano naturalmente a deformazioni meno accentuate. In esse l'asse maggiore è orientata pressochè normalmente alla linea di valle, mentre la minore accenna ad una contrazione. Questo fatto consente un'interpretazione $\mathrm{di}$ notevole interesse, della quale parlerò più oltre.

\section{MASSIMA DISTORSIONE.}

I valori massimi della distorsione, secondo la definizione che ne è stata data nel testo, sono rappresentati, per quanto si riferisce agli spostamenti osservati passando dalla prima alla seconda triangolazione, nelle tav. III $a$ ).

Allo scopo di ridurre al minimo l'esposizione, riassumerò nelle pagine che seguono, i risultati dell'indagine relativi alle restanti triangolazioni, tenendo presente che si esprimono le variazioni subite dalle grandezze in esame nel passare da una triangolazione alla successiva.

\section{Dilatazione}

Triangolazione III-II: come risulta dalla tav. I $b$ la dilatazione nel passaggio da una all'altra triangolazione, ha subito un cambiamento di segno dal positivo al negativo, il che sta a significare che nella zona si è verificata una contrazione. (tav. I $b$ ).

Triangolazione IV-III: nel passaggio dalla III alla IV il fenomeno si inverte: nella zona interessata si verifica cioè una distensione (dilatazilne positiva), (tav. Ic).
Triangolazione V-IV: nuova inversione del segno corrispondente a contrazione.

Triangolazione VI-V: la dilatazione torna ad essere positiva fatta esclusione di una zona molto ristretta intorno al baricentro del triangolo [7] [18] [19], che anche per il resto si è generalmente dimostrata anomala.

Triangolazione VII-VI: nel passaggio dalla VII alla VI triangolazione, il comportamento della dilatazione non è stato univoco, nel senso che ha alternato zone con dilatazione positiva a zone caratterizzate da contrazione, sebbene le prime prevalgono sulle seconde.

Triangolazione VIII-VII: in quest'ultimo passaggio la dilatazione è tornata ad essere decisamente negativa (contrazione).

\section{DISTORSIONE.}

L'andamento della distorsione si presenta meno semphce; e ciò è naturale, se si pensa alla sua definizione.

Il giuoco degli scorrimenti è strettamente legato all'ampiezza degli spostamenti e alla loro variazione: ecco perchè - a differenza di quanto si verifica per la dilatazione - in ogni tavola le zone caratterizzate da distorsioni positive si alternano a zone con distorsioni di segno contrario.

Da una triangolazione all'altra le zone possono conservare approssimativamente le stesse estensioni, mutando di segno; ma questo può anche verificarsi, a seconda dell'andamento delle variazioni, che influiscono decisamente nell'alternanza dei segni.

\section{Rotazione}

A differenza della distorsione, questa grandezza risente meno delle variazioni dell'ampiezza degli spostamenti, quanto dell'inversione, del senso del movimento.

Poichè da una triangolazione all'altra il segno dei movimenti generalmente si inverte, lo stesso avviene per la rotazione.

\section{DEFORMAZIONI PRINCIPALI.}

È interessante osservare come l'andamento delle ampiezze (di distensione e di con- 
trazione) delle deformazioni principali sia strettamente collegato all'andamento della dilatazione, positiva o negativa, tenuto debito conto della distorsione.

Le tavole annesse appaiono al riguardo così chiare, che ritengo superflue ulteriori delucidazioni.

Come si è già detto per la dilatazione, le linee a tratto continuo rappressentano le distensioni mentre quelle tratteggiate, le contrazioni.

\section{PARTE TERZA}

\section{CONCLUSTONI.}

Va premessa un'osservazione d'ordine generale. I valori riportati nelle tabelle, e tradotti in grafico nei diagrammi - attese la complessità del terreno, a punti del quale le misure si riferiscono, e la schematizzazione d'altronde inevitabile, a cui sono dovuta ricorrere nei calcoli - hanno limiti d'incertezza non facilmente calcolabili; comunque, talvolta certamente sensibili. L'attendibilità delle misure geofisiche e - forse più ancora _ di quelle geodetiche, presenta oscillazioni, intorno alla media, sovente dell'ordine dei valori osservati. Quel che però conta in questo genere di indagini è essenzialmente l'andamento generale del fenomeno, la direzione di variazione delle grandezze in giuoco, fatta astrazione dai limiti di certezza.

Dai risultati del presente studio possono trarsi conclusioni, alcune delle quali - a mio avviso - consentono di mettere in luce aspetti ignorati delle perturbazioni, comunque collegate all'esistenza di un bacino idroelettrico, e di chiarirne altri a torto trascurati.

In primo luogo, va messo in rilievo che ogni triangolazione è caratterizzata - generalmente - da un solo segno (positivo o negativo) per la dilatazione. Ciò sta a significare che, in tutta la zona interessata dalla diga e dal bacino idrico (almeno nella parte sottoposta a misure geodetiche) si ha contemporaneamente o espansione del mezzo (dilatazione positiva) o contrazione. Il variare dell'intensità o della direzione di questi fenomeni dà luogo a distorsioni, più o meno accentuate. Gli assi principali delle ellissi di deformazione (tese o contratte) esprimono - con le loro lunghezze e direzioni l'entità della deformazione elastica e l'azimut secondo cui agisce.

L'azione dell'invaso e quella termica sulla diga non sono sufficienti a spiegare l'andamento dei fenomeni ossr rvati. $\grave{E}$ necessario dare il debito peso anche all'azione termica stagionale sulla valle, pre $x$ nel suo insieme.

L'azione termica sul calcestruzzo è già stata ripetutamente osservata e calcolata nei suoi effetti. Del resto, è noto dai manuali di costanti fisiche, che per il cemento e il calcestruzzo, il coefficiente di espansione lineare è dell'ordine di $10-14 \times 10^{-6}$. Ma non è affatto trascurabile, nei suoi confronti, il coefficiente per rocce in genere. Risulta infatti dell'ordine di $4 \times 10^{-6}$ quello del marmo, di 8,3 quello del granito, variante fra 7 e 12 quello dell'arenaria, ecc. Si potrà osservare che la vegetazione e il terreno sciolto di copertura possono alterare l'azione termica sulla roccia sottostante; però, non potranno mai annullarla. Ricerche fatte sull'argomento provano che la conducibilità termica (quantità di calore che attraver'sa l'unità di superficie nell'unità di tempo, per differenza unitaria di temperatura fra due superficie distanti l'unità) per vari tipi di terreni è tutt'altro che trascurabile e che è sensibile fino alla profondità di qualche metro. Spessori di terreni sciolti potranno quindi ritardare ed attenuare l'azione termica sulla roccia sottostante, senza però annullarla; specie nella zona in questione dove, a vasti tratti di roccia scoperta, si alternano zone ove il terreno ricoprente la roccia presenta spessori di pochi decimetri.

Già in studi precedenti $\left({ }^{2}\right)$, era stata osservata un'influenza stagionale sull'apertura della valle. Si veda ad es. la fig. 4. In essa sono rappresentate, fra l'altro, le variazioni angolari osservate a Monte Ricco (Pieve di Cadore), sovrastante il bacino idrico in spalla destra e sulla sponda destria del Piave, immediatamente a valle della diga, dal 4-XI al 23-XII-1950: sono variazioni angolari conseguenti ad allargamento della valle, per contrazione termica della roccia.

È chiaro che la contrazione varierà, a parità di altre condizioni, con l'inclinazione 
della roccia sull'orizzonte. Nel caso in esame, essa è andata certamente crescendo con l'altezza: basta ricordare infatti che il sottostante bacino, almeno nelle sue acque profonde, conservava una temperatura dell'ordine di 4 ' $\mathrm{C}$, mentre all'esterno la temperatura raggiungeva punte di 10 e più gradi sotto zero. Da qui un'azione differenziale crescente virso l'alto, rivelata dai clinografi.

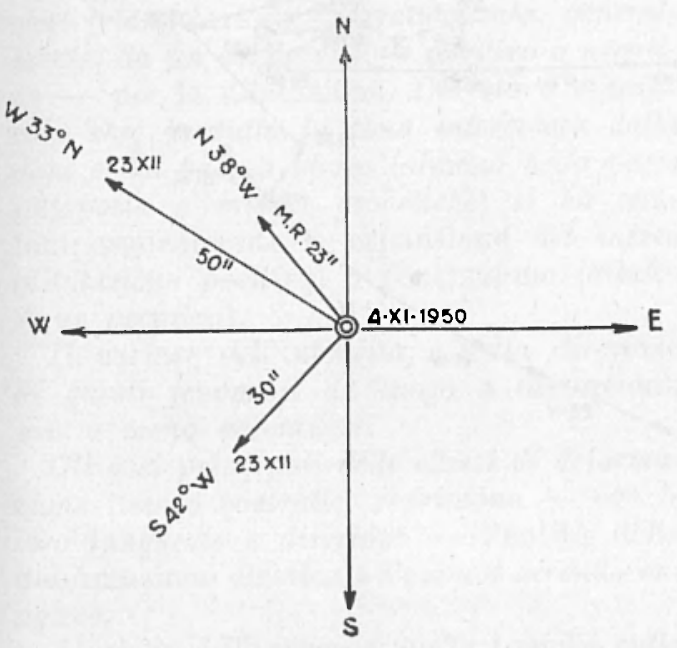

Fig. 4. - Variazioni angolari osservate a Monte Ricco

Ma la fig. 4), non è la sola testimonianza a favore di quanto si afferma. Sempre in quella relazione della SADE sopra citata, è riportato l'andamento dei movimenti angolari osservati nel concio XXIX (prossimo alla spalla destra) da un invaso allo svaso ${ }^{\top}$ successivo. Riportiamo qui, col numero di successione 5), detta figura.

Il rapido riempimento del bacino (70 e più metri di invaso dal 5 a fine maggio 1950) spiega la netta flessione verso valle (con chiara componente verso la spalla destra) osservata dal 5 al $20-V$. Dal $2-V$ al 18-IX si manifestano contemporaneamente l'azione termica (in fase positiva) con tendenza a riportare la diga verso monte $\mathrm{e}$ verso sinistra, contrastata dall'azione dell'invaso, ora in lenta salita - escluso il mese di Luglio, con invaso a quota costante, e durante il quale si ha una lieve flessione, prevalentemente verso monte.
Dalla fine di Agosto la flessione verso sinistra si compone con lieve crescente flessione verso valle. Alla fine di Ottobre la flessione è decisamente verso valle, mentre si inverte la flessione spalla-spalla, ora con tendenza verso destra. Dai primi di Ottobre alla fine di Dicembre la flessione verso destra è decisa ed esclusiva. Poi, con qualche contrasto, riprende la flessione verso monte e quindi, con l'Aprile, con netta prevalenza verso sinistra.

Per le deduzioni che seguono, si deve ricordare che il concio XXIX è uno dei conci marginali verso la sponda destra.

I fenomeni descritti non si possono spiegare limitando le considerazioni all'azione termica sulla diga. Se cosi fosse - e l'azione termica sul sistema roccioso a cui la diga è ancorata fosse trascurabile - un aumento di temperatura fletterebbe la parte centrale della diga a monte - come effettivamente flette - ma i conci marginali dovrebbero flettere verso le rispettive sponde e il contrario dovrebbe verificarsi nel caso di variazione termica in senso negativo. Nella realtà, mentre i conci centrali flettono effettivamente verso monte 0 verso valle, a seconda di un aumento o di una diminuzione di temperatura, contemporaneamente i conci marginali flettono verso il centrovalle o verso l'esterno. Nel caso in esame, il concio XXIX flette verso sinistra per aumento e verso destra per diminuzione di temperatura. La spiegazione mi sembra chiara; nel primo caso, si verifica una dilatazione della roccia in spalla destra, con spinta dei conci marginali a sinistra, nel secondo caso, la contrazione della roccia attira a sé i conci marginali, che fletteranno a destra. E quello che indica la fig. 5. Nei periodi di inversione termica, l'azione dell'invaso, prevale con flessioni verso valle o verso monte, a seconda dei casi,

Si potrà obiettare che non vi è contemporaneità fra $i$ fenomeni di flessione e le variazioni termiche nell'aria. $\mathrm{Ma}$ anche ciò si spiega: è stato provato infatti che l'onda termica nei solidi è sempre in ritardo, che pur variando con la natura del solido, può raggiungere un mese e mezzo o due per profondità di qualche metro. Ciò spiega i ritardi osservati.

Un'ulteriore prova di quanto qui si affer- 
ma risulta da un altro aspetto del fenomeno: quello geodetico.

A valle della diga, a sufficiente distanza dal bacino idrico per non subire le conseguenze delle continue sue variazioni, agiscono essenzialmente le varia-
Qualche netta conferma a quanto è stato sopra riportato, si ha pure nei movimenti delle sponde del bacino, osservati per via geodetica: particolarmente importante quella fra la $9^{a}$ e la $10^{a}$ triangolazione (v. prospetto V, pag. 7 della "Seconda relazione

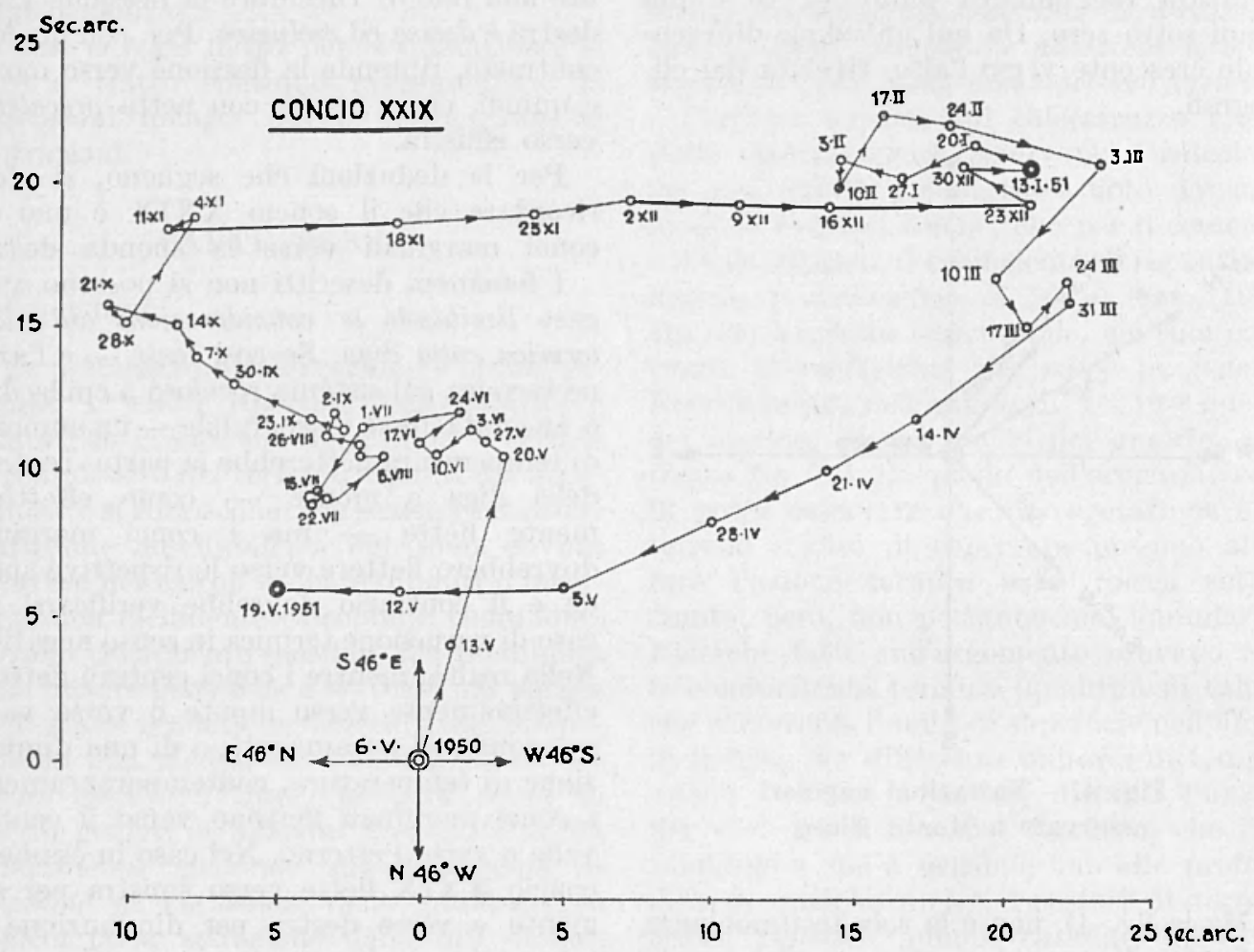

Fig. 5. - Variazioni angolari osservate nel Concio XXIX della diga di Pieve di Cadore.

zioni termiche. Se si esaminano p. es. gli spostamenti osservati nella postazione 6 (fig. $A$ ), e si tien conto delle epoche di osservazione, si nota che la direzione degli spostamenti è pressoché normale alla linea di fondo valle, e il senso quello che generalmente ci si deve aspettare, sulla base dell'andamento termico. La sponda sinistra, del resto, si trova - in quella zona - molto meglio esposta all'insolazione della sponda destra, molto incassata e boscosa.

E chiaro che l'azione termica si riflette pure sui caposaldi della base.

Nella zona interessata dal bacino, l'azione termica viene naturalmente alterata dalle variazioni di livello. sulle misure geodetiche ", Relazioni e Studi, 3 Marzo 1955 - S. A. D. D.).

Quanto è stato qui esposto, presuppone l'azione perturbante di due sole cause agenti: quella termica e quella dell'invaso. E noto però che, in effetti, altre cause di natura geologico - geofisica, accidentali o periodiche (a breve periodo o di estensione secolare) possono sovrappore le loro contemporanee azioni: lenti: moti bradisismici, movimenti relativi di blocchi, o ammassi rocciosi, adiacenti, ecc. Talvolta queste ultime azioni possono essere prevalenti, così da mascherare o rendere in apparenza contradittorie quelle sopra accennate. Fatti del genere furono constatati anche a Pieve, specie durante il 1953. 


\section{RIASSUNTO}

Dai risultati del presente studio possono trarsi conclusioni, alcune delle quali consentono di mettere in luce aspetti ignorati delle perturbazioni, comunque collegate all'esisten$z a$ di un bacino idroelettrico, e di chiarirne altri a torto trascurati.

In primo luogo, va messo in rilievo che ogni triangolazione è caratterizzata, generalmente, da un solo segno - positivo o negativo - per la dilatazione. Ciò sta a significare che, in tutta la zona interessata dalla diga e dal bacino idrico (almeno nella parte sottoposta a misure geodetiche) si ha contemporaneamente o espansione del mezzo (dilatazione positiva) o contrazione (dilatazione negativa).

Il variare dell'intensità e della direzione di questi fenomeni dà luogo a distorsioni, più o meno accentuate.

Gli assi principali delle ellissi di deformazione (tese o contratte) esprimono - con le loro lunghezze e direzioni - l'entità della deformazione elastica e l'azimut secondo cui agisce.

L'azione dell'invaso e quella termica sulla diga non sono sufficienti a spiegare l'andamento dei fenomeni osservati. $\mathrm{E}$ necessario dare il debito peso anche all'azione termica stagionale sulla valle, presa nel suo insieme.

Quanto è stato esposto, presuppone l'azione perturbante di due sole cause agenti: quella termica e quella dell'invaso. Si sa, però, che in effetti altre cause di natura geologicogeofisica, accidentali o periodiche (a breve periodo o di estensione secolare) possono sovrapporre le loro contemporanee azion $i$ : lenti moti bradisismici, movimenti relativi di blocchi, o ammassi rocciosi adiacenti, ecc. Talvolta queste ultime azioni possono essere prevalenti, cosi da mascherare o rendere in apparenza contradditorie quelle sopra accennate.

\section{ABSTRACT}

From the result of this study several conclusions may be drawn, some of which allow to enlighten unknown aspects of the perturbations which are however connected with the existence of an hydroelectric reservoir; they allow further to make clear other wrongfully neglected aspects.

It should be first remarked that any triangulation is generally characterized by only one symbol (positive or negative) for the dilatation. This means that, in the whole territory which is interested on the dam and reservoir (at least in the part of it which has been subjected to geodetic measurements), it may occur contemporarily an expansion (positive dilatation) or a contraction of the means. The variation of intensity or direction of these phenomena engenders a more or less emphasized distortion. The main axes of the deformation ellipses (stretched or contracted) express, by their lengths and directions, the entity of the elastic deformation and the azimut by which it acts.

The action of the hydraulic-reservoir and the thermic action of the dam are not sufficient to explain the process of the noticed phenomena. Adequate attention must be given to the seasonal thermic action on the valley, considered in its whole. The matter which has been exposed hitherto, presupposes the disturbing action of only two worling factors: the thermic action and the action of the hydraulic-reservoir. It is known, in effect, that other geological or geophysical agents, accidental or periodical (with a short period or a centurylong span), may superpose their contemporary actions: slow bradiseismic motions, corresponding motions of blocks or a adjoining rocky masses, etc. Sometimes, the latter may be so prevailing, as to disguise or render seemingly contradictory the actions mentioned above. 
Dal 16 al 24 settembre 1958 avrà luogo a Chamonix (Monte Bianco) un Symposium indetto dalla Commissione delle nevi e dei ghiacciai dell'Associazione Internazionale d'Idrologia Scientifica.

Il tema proposto è il seguente:

- gli aspetti fisici del movimento dei ghiacciai.

Gli studiosi che vorranno partecipare al
Symposium dovranno inviare al Presidente della Commissione d'Idrologia Scientifica (presso il Ministero dei Lavori Pubblici Servizio Idrografico Centrale - Roma) entro il $1^{\circ}$ maggio 1958 le memorie in duplice copia.

Per qualsiasi altra informazione inerente alle condizioni di soggiorno e al viaggio rivolgersi al Ministero dei Lavori Pubblici Servizio Idrografico Centrale - Roma.

Direttore: Prof. Enrico Medi

Prof. Pietro Caloi - Responsabile

Tipografia Pio X - Roma - Via degli Etruschi, 7 - 20-VII-58 

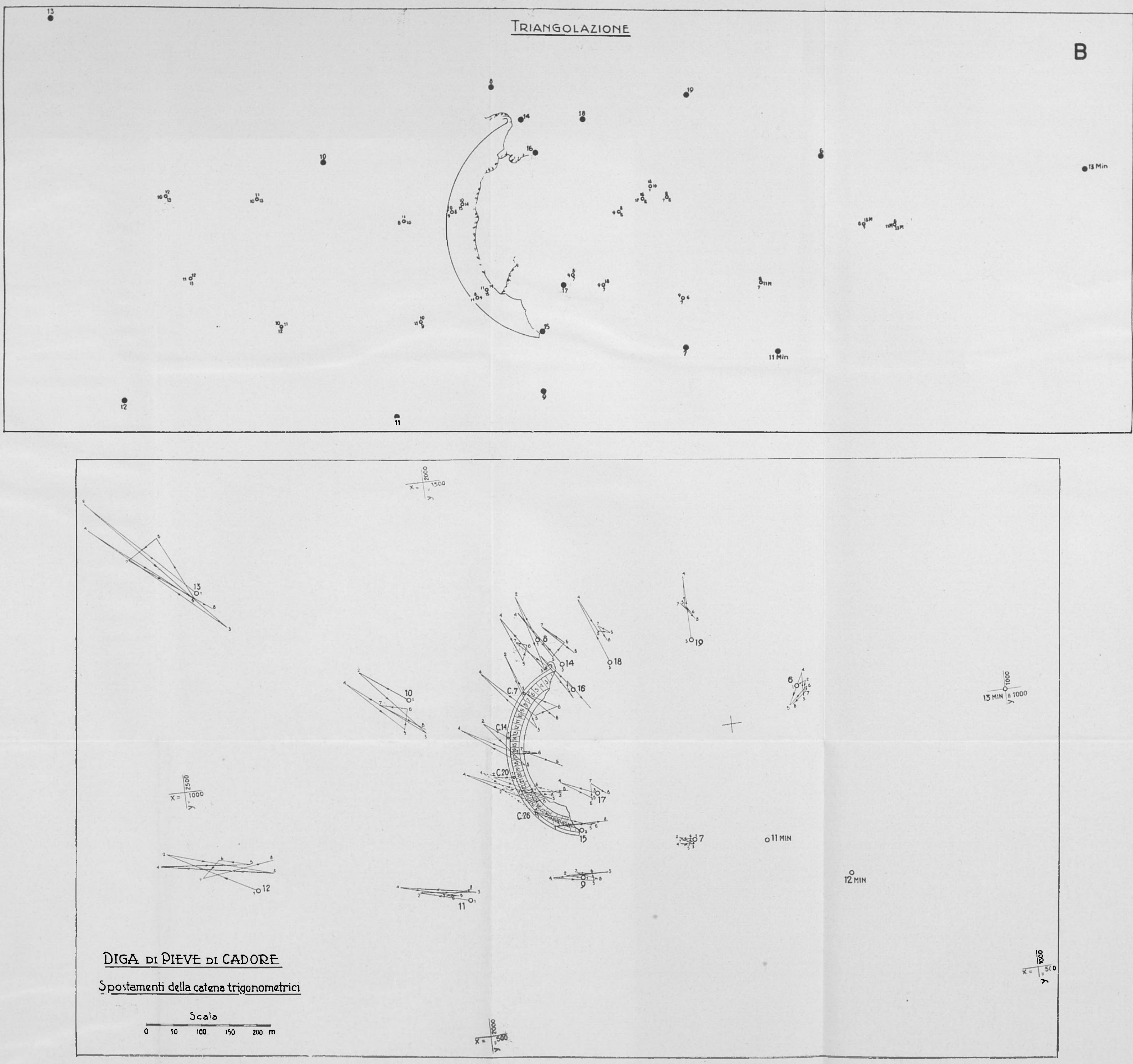

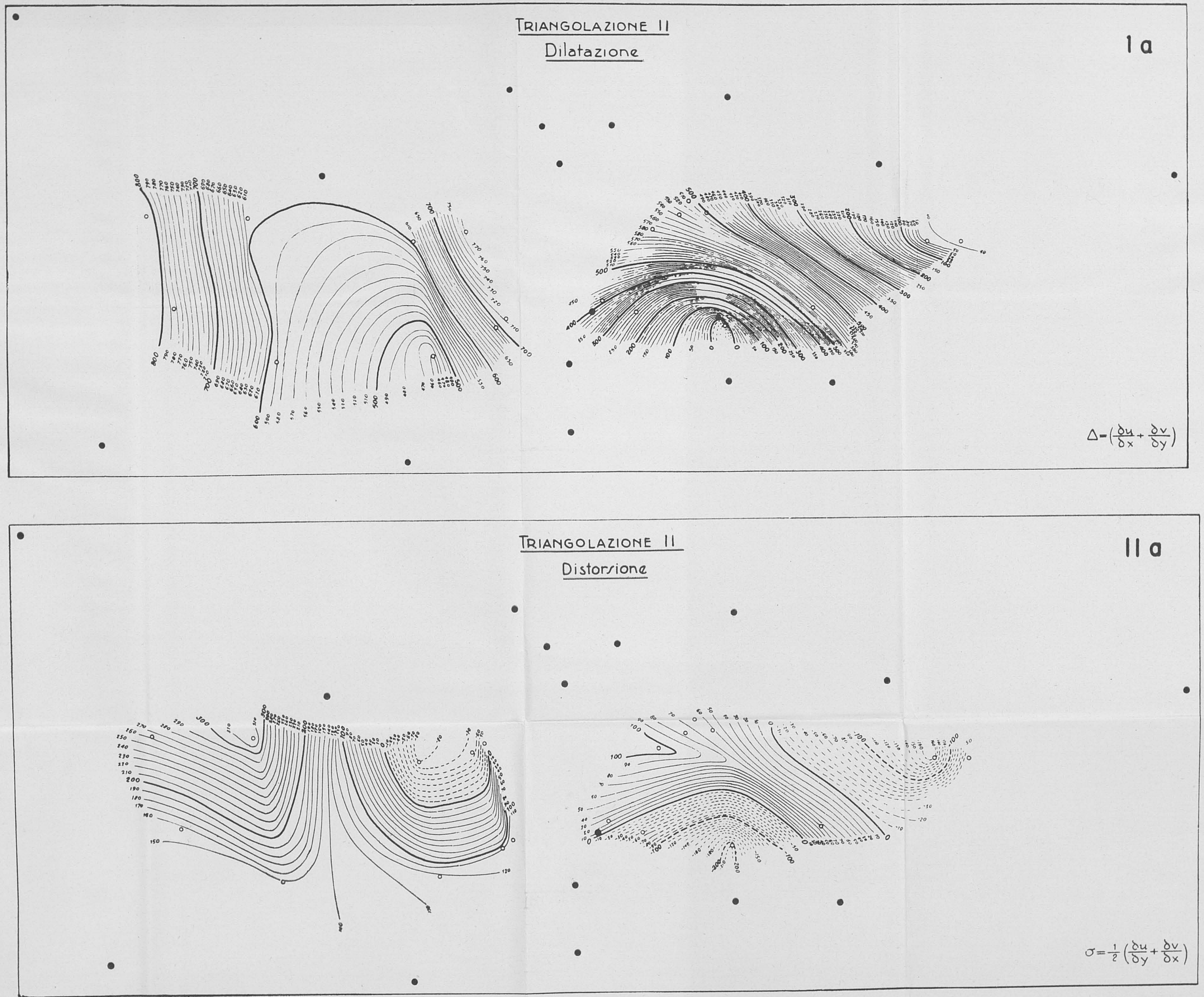

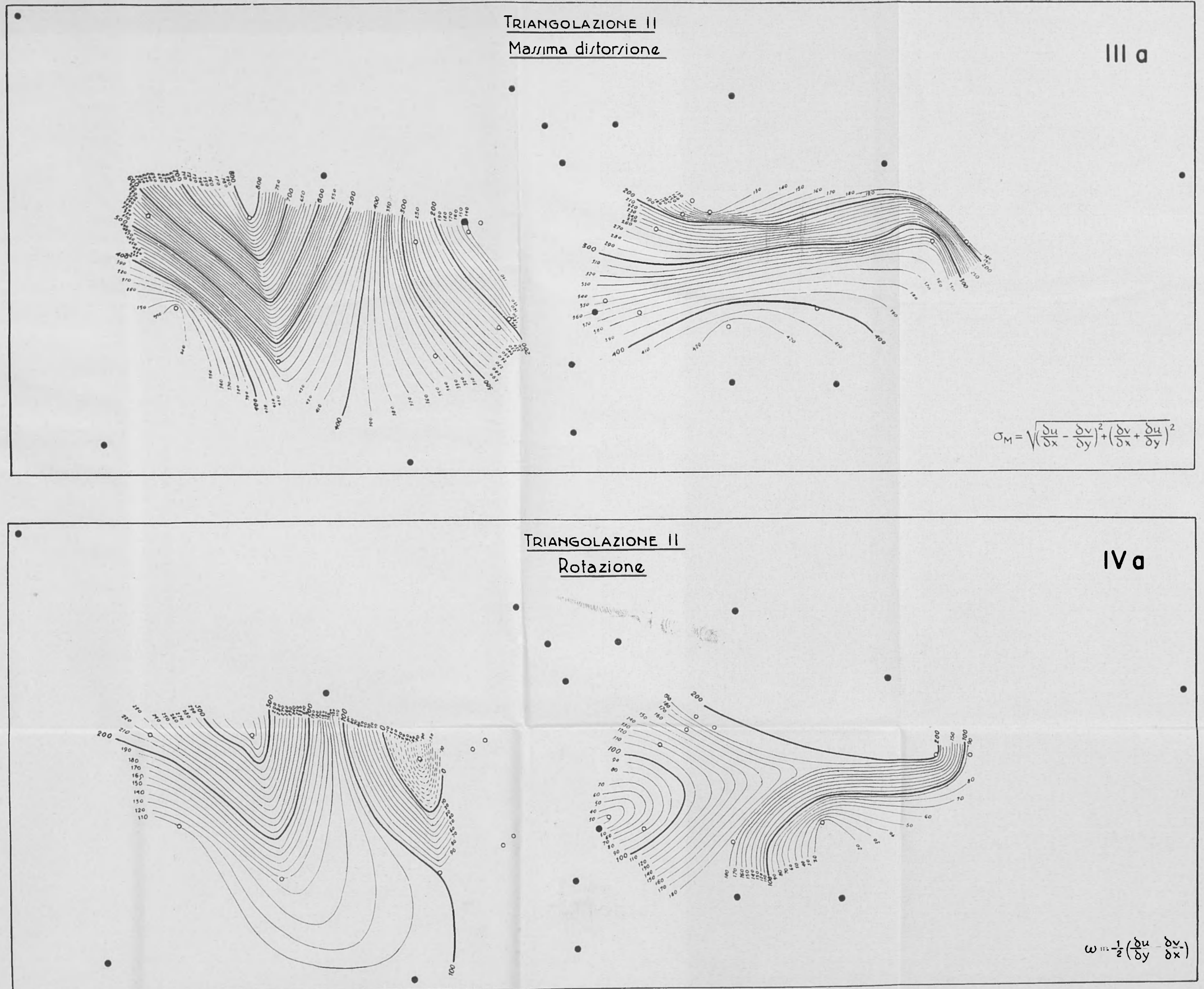


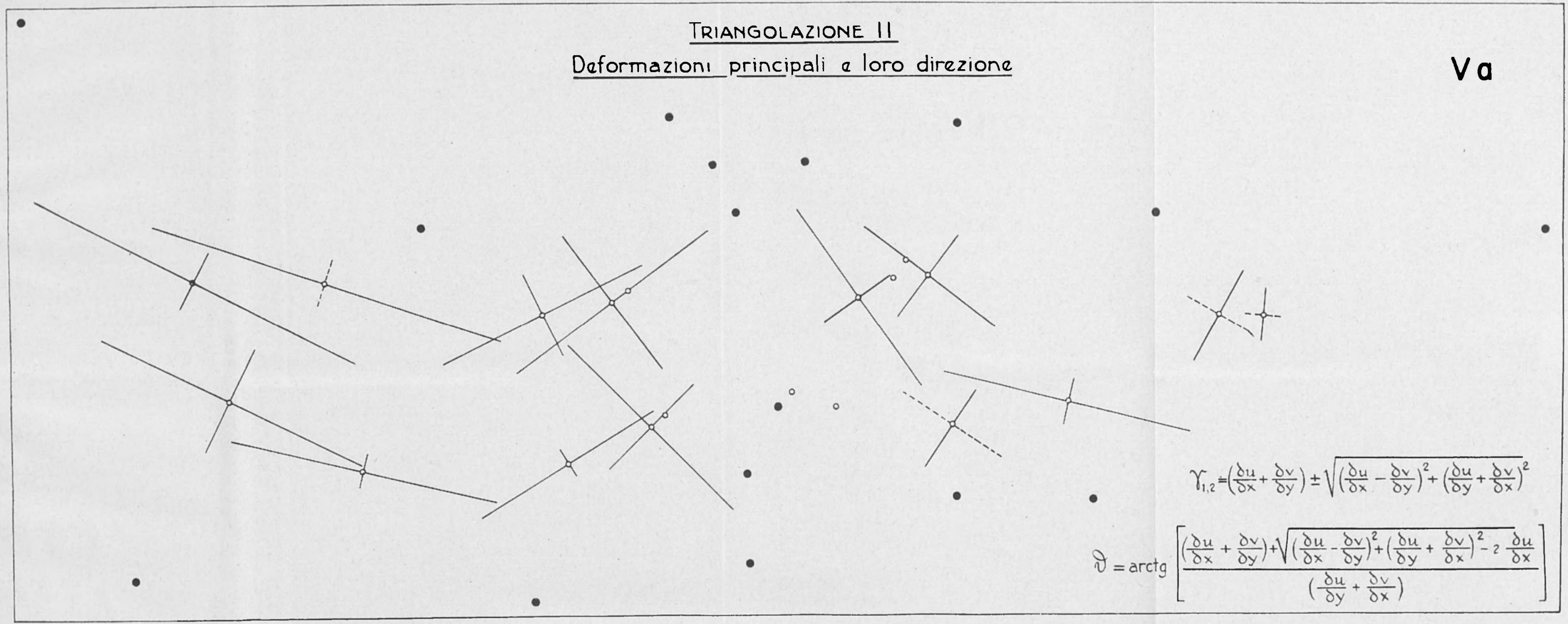



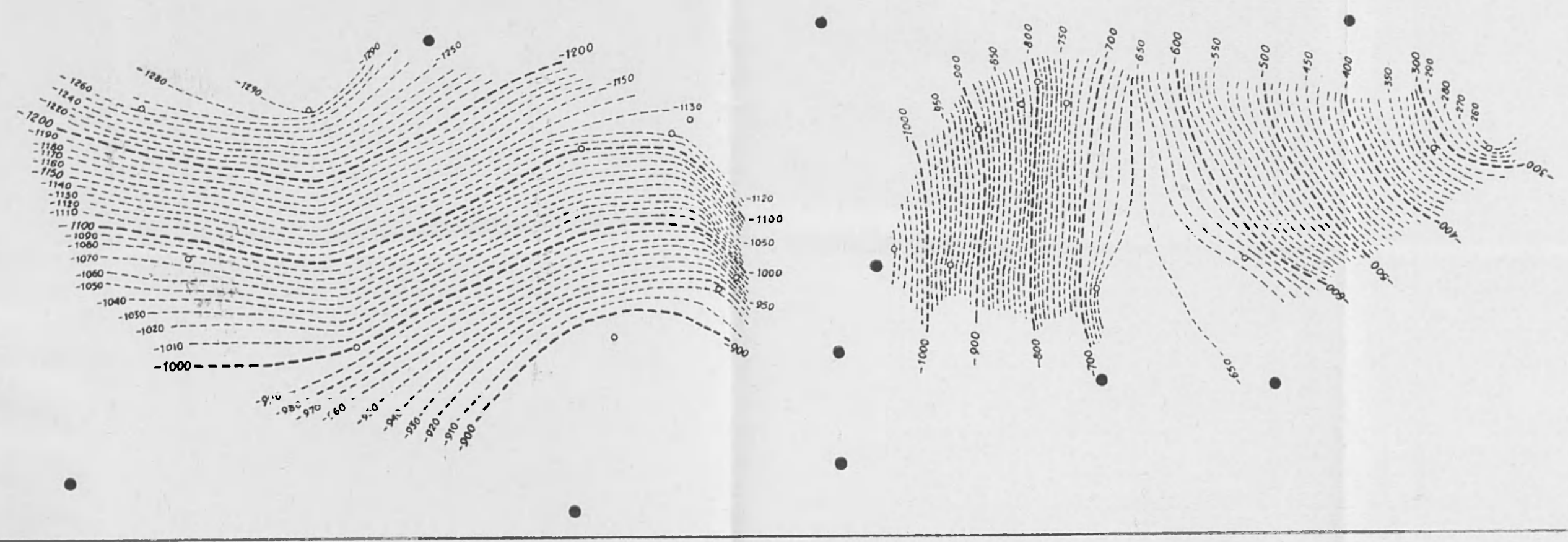

\section{TrIANgolazione IV}

Dilatazione

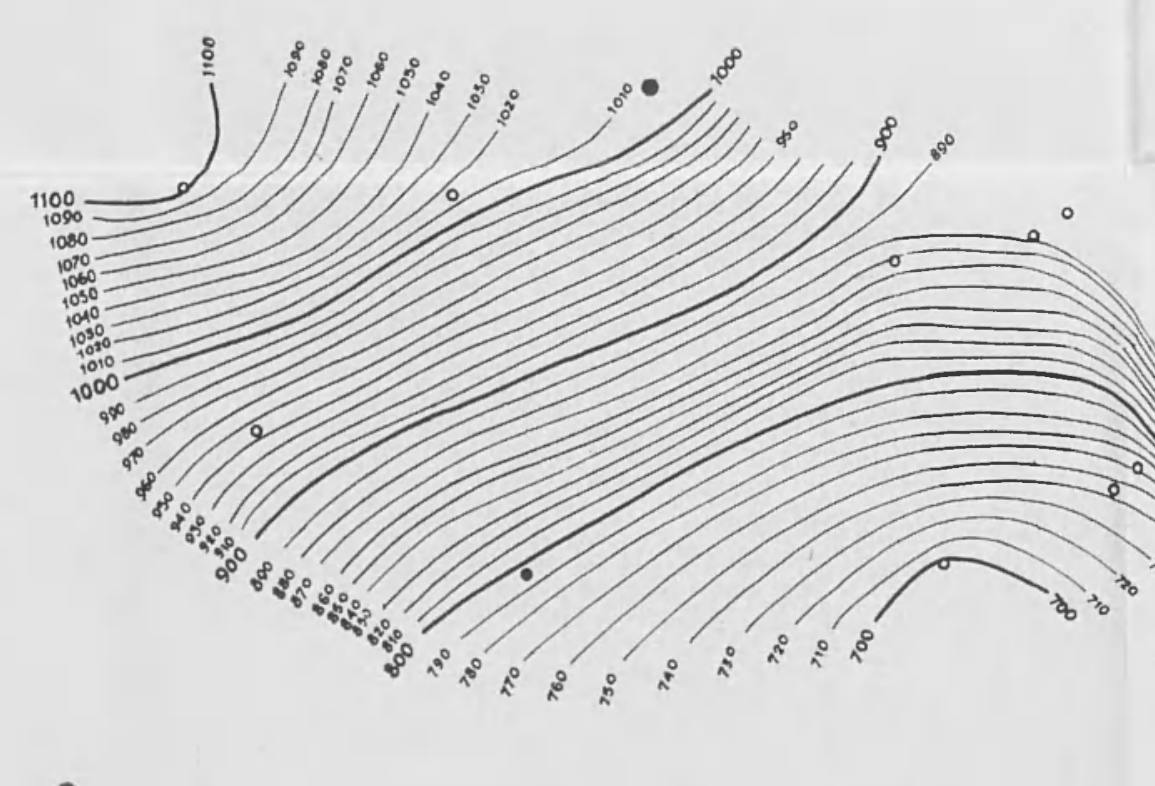

-

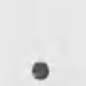

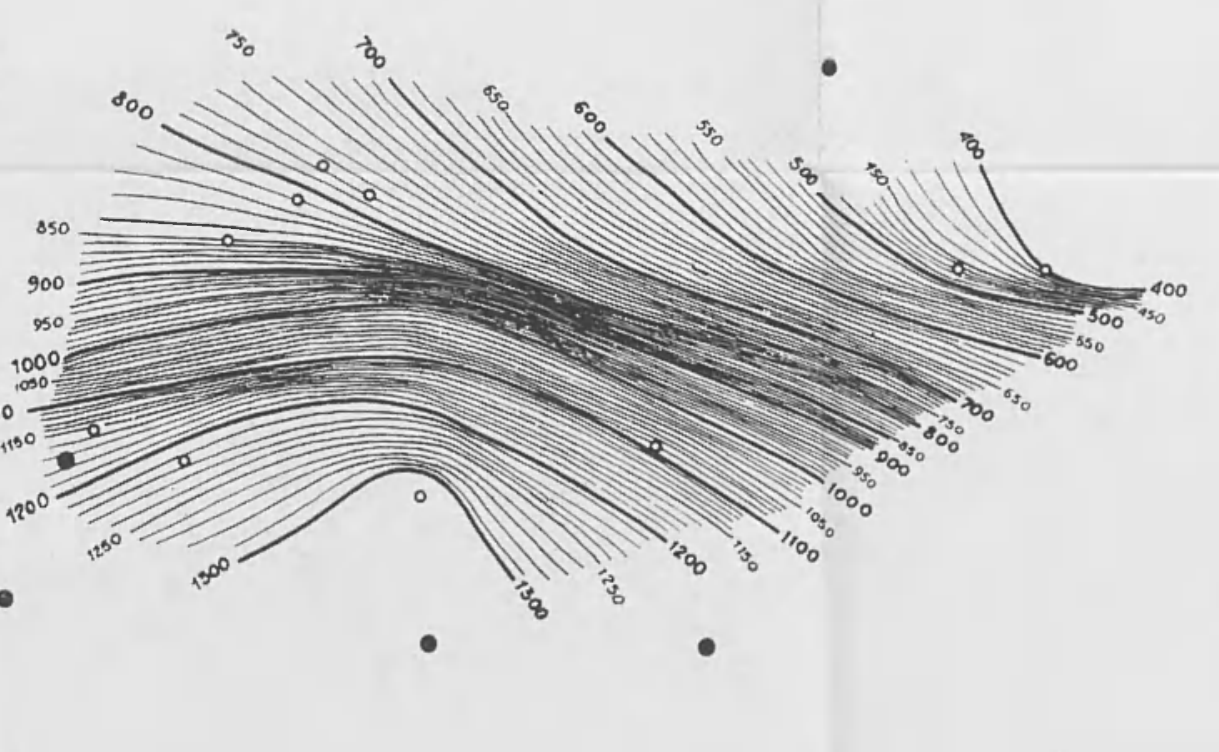



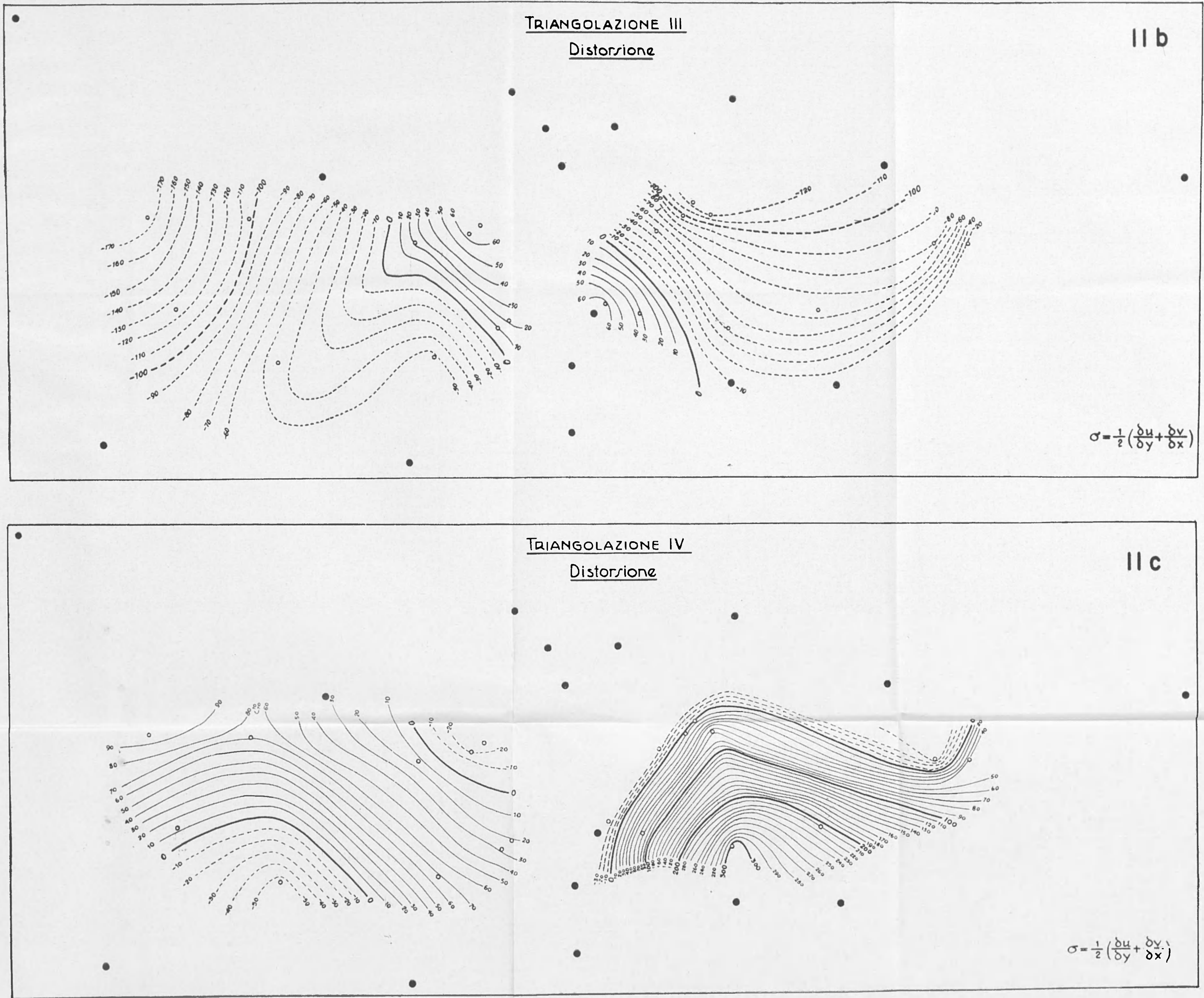

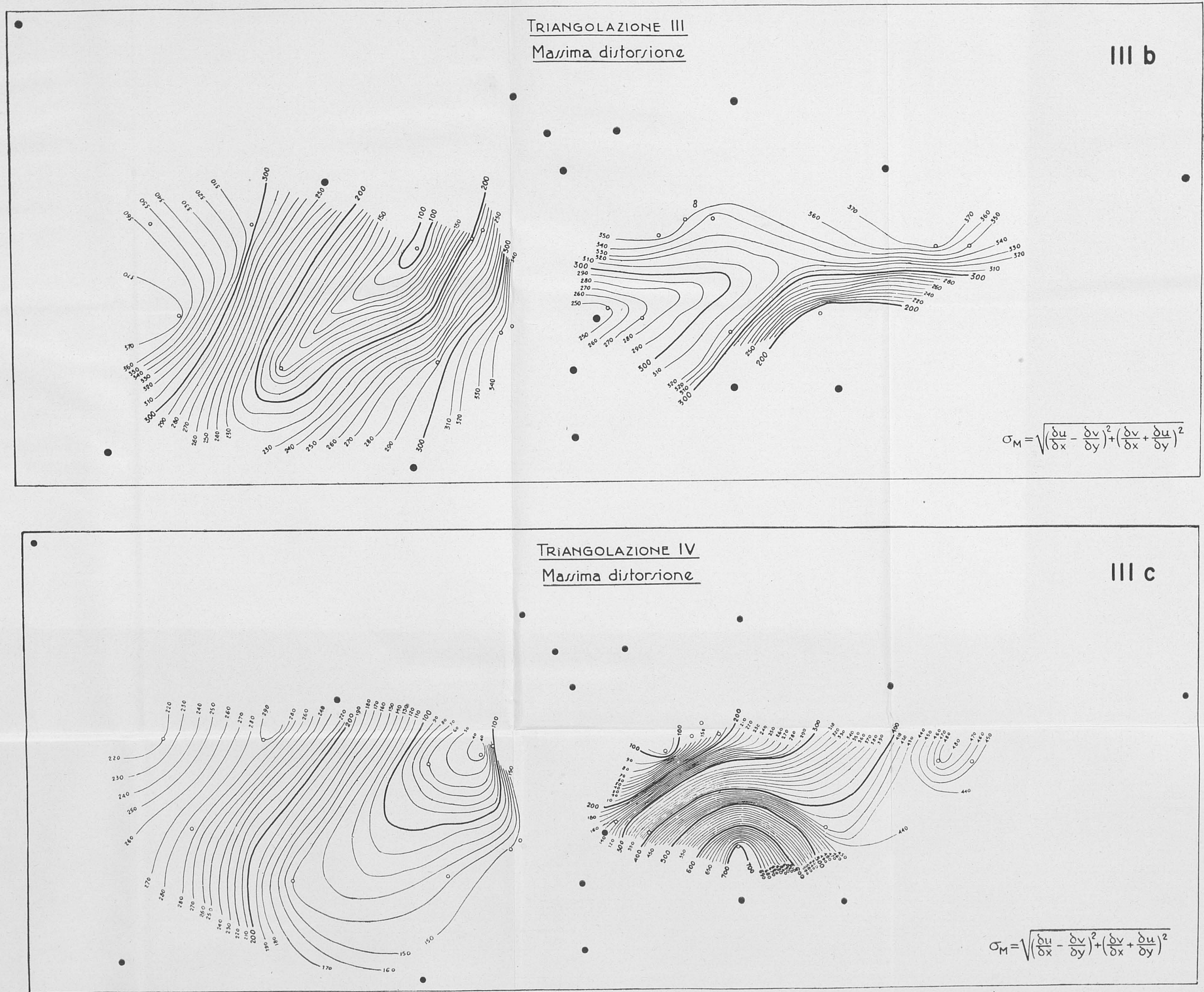
-

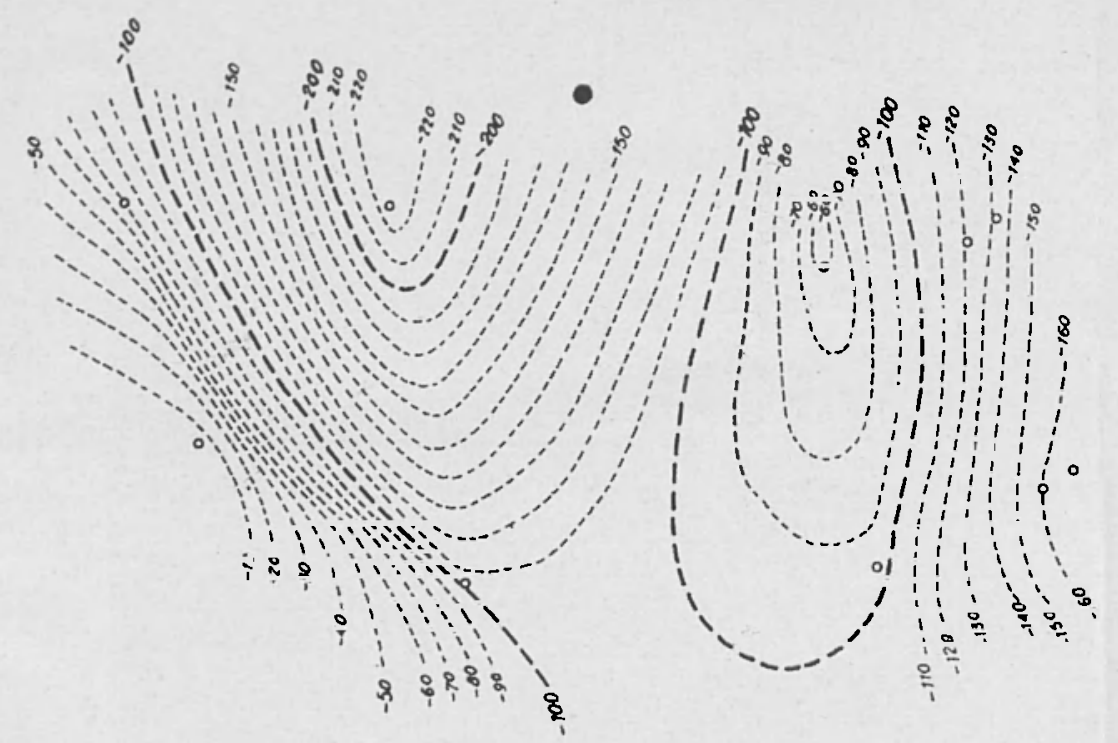

-
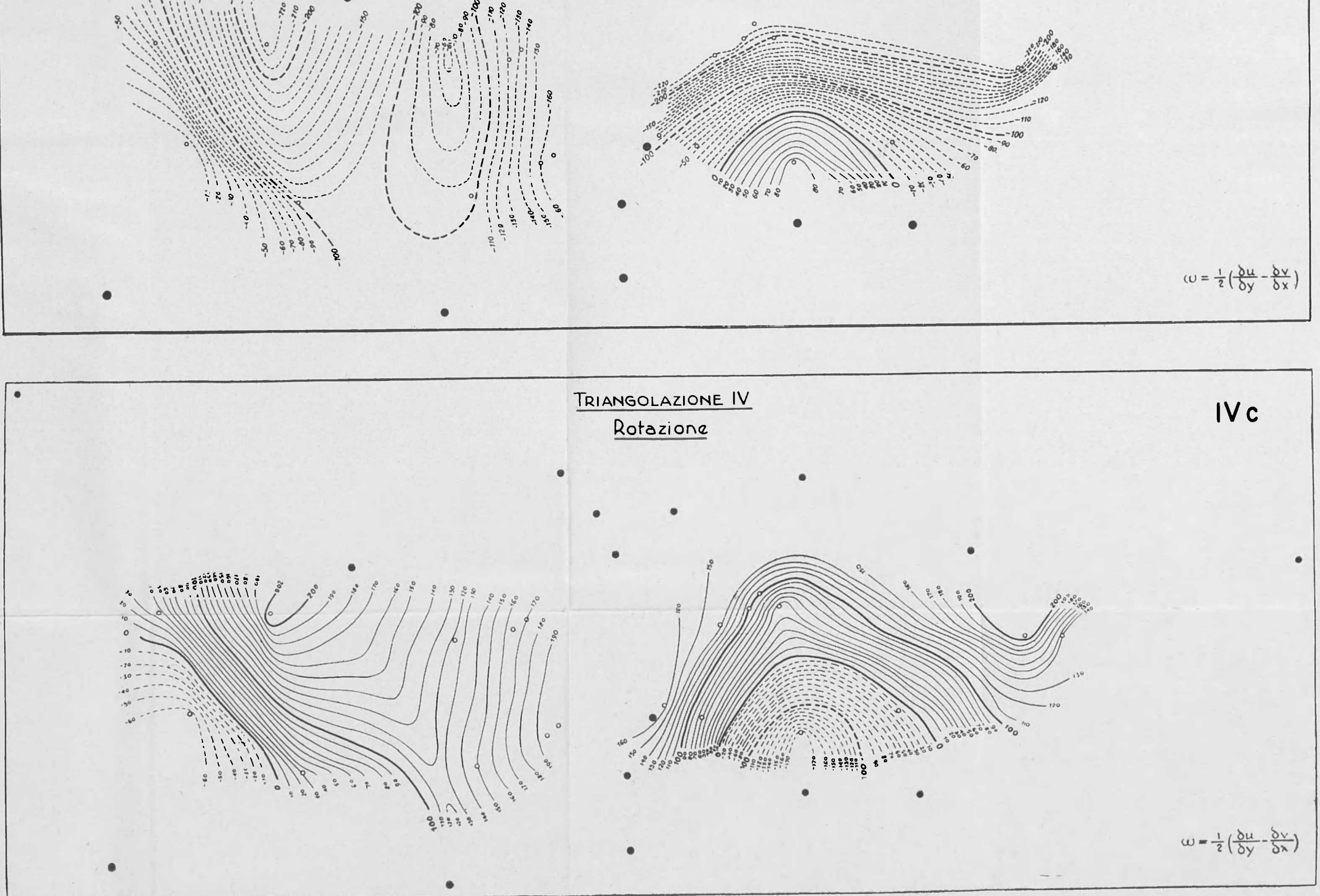
TRIANGOLAZIONE III

Deformazioni principali e loro direzione

V b

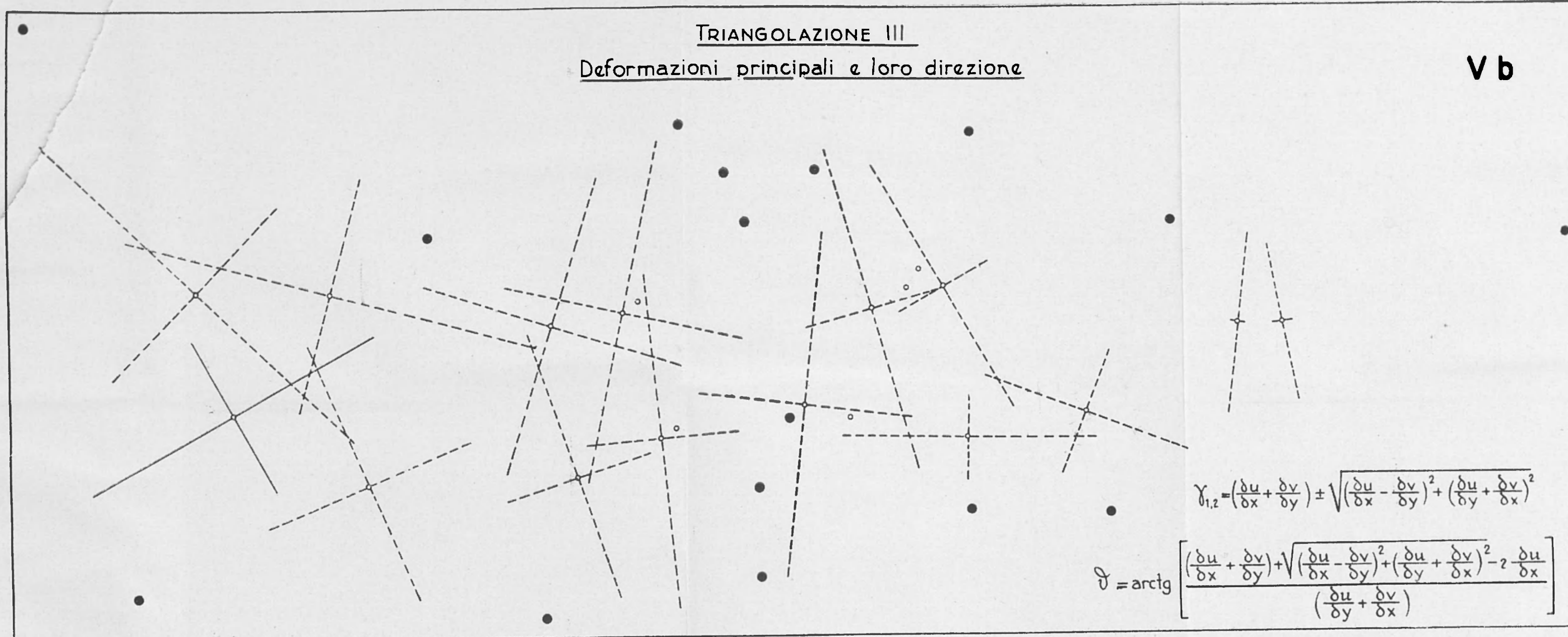

TRIANGOLAZIONE IV

Deformazioni principali e loro direzione

Vc

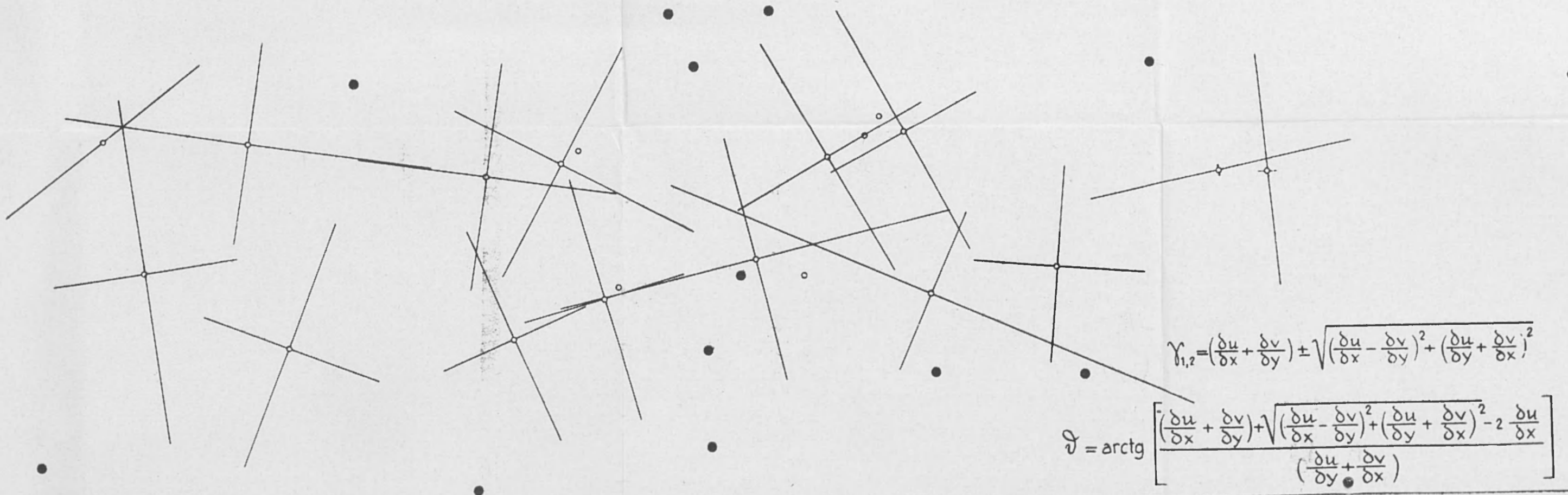

